\title{
A Fourier transform spectroradiometer for ground-based remote sensing of the atmospheric downwelling long-wave radiance
}

\author{
Giovanni Bianchini, Francesco Castagnoli, Gianluca Di Natale, and Luca Palchetti \\ Consiglio Nazionale delle Ricerche, Istituto Nazionale di Ottica, Via Madonna del Piano 10, 50019 Sesto Fiorentino, Italy \\ Correspondence: Giovanni Bianchini (giovanni.bianchini@ino.cnr.it)
}

Received: 13 July 2018 - Discussion started: 25 July 2018

Revised: 25 December 2018 - Accepted: 3 January 2019 - Published: 30 January 2019

\begin{abstract}
The Radiation Explorer in the Far Infrared - Prototype for Applications and Development (REFIR-PAD) is a Fourier transform spectroradiometer that has been designed to operate from both stratospheric balloon platforms and the ground. It has been successfully deployed in a stratospheric balloon flight and several ground-based campaigns from high-altitude sites, including the current installation at the Italian-French Concordia Antarctic station. The instrument is capable of operating autonomously with only a limited need of remote control and monitoring and provides a multiyear dataset of spectrally resolved atmospheric downwelling radiances, measured in the $100-1500 \mathrm{~cm}^{-1}$ spectral range with $0.4 \mathrm{~cm}^{-1}$ resolution and a radiometric uncertainty of better than $0.85 \mathrm{~mW}\left(\mathrm{~m}^{2} \mathrm{srcm}^{-1}\right)^{-1}$.
\end{abstract}

\section{Introduction}

The measurement of the atmospheric downwelling longwave radiance (DLR) is a crucial task in climate and Earth radiation budget (ERB) studies since it provides the complementary quantity to the top-of-atmosphere outgoing longwave radiance (OLR) measured from space. The knowledge of both these quantities is needed in order to achieve a complete characterization of the ERB (Wild et al., 2013).

Unfortunately, for ground-based measurements, it is very difficult to achieve a global coverage because DLR measurements can be performed only from limited locations above land areas (Ohmura et al., 1998), thus causing large errors in the estimation of the global balance of energy fluxes. This uncertainty limits our ability to identify with sufficient reliability the response (feedback) of the Earth's climate to the variation in different components (forcing) (Stephens et al., 2012).

Nevertheless, some new insights can be obtained by using spectrally resolved measurements (Huang et al., 2007; Huang, 2013). A spectrally resolved measurement of the DLR provides significant advantages with respect to spectrally integrated measurements, allowing for an accurate identification of the radiative forcing and feedback signatures, and thus the contributions to the ERB, of the various atmospheric constituents (Gero and Turner, 2011).

Conversely, compared to standard DLR broadband integrated measurements providing the downwelling irradiance at ground level, such as those from the Baseline Surface Radiation Network (BSRN) (Ohmura et al., 1998), spectrally resolved measurements typically measure only the radiance for a single line of sight and in a small solid angle. Further calculations, or several measurements made at different angles, are needed to estimate the irradiance. This limitation is typically present for space measurements for which the OLR irradiance is calculated from the observation of a few lines of sight from polar orbit, e.g., CERES (Loeb et al., 2005, 2007), or from a single line of sight from geostationary orbit, e.g., GERB (Clerbaux et al., 2003).

This limit can be overcome with the use of a radiative transfer model and the application of an inversion procedure on the measured atmospheric emission spectra to retrieve vertical profiles of variables such as water vapor, temperature, and minor constituents, which are relevant for the calculation of DLR. In practice these variables can be used in the forward model to reconstruct radiance in the lines of sight that were not directly measured (Palchetti et al., 2017) and thus calculate the downwelling irradiance in clear sky conditions. This approach has also been applied to satellite obser- 
vations to derive CERES fluxes from IASI spectral measurements (Turner et al., 2015).

Spectral observations in the thermal infrared have been used to retrieve atmospheric state from both top-ofatmosphere (see, e.g., Ridolfi et al. (2000)) and ground-based observations (Smith et al., 1999) and to perform radiative closure experiments (Turner et al., 2004; Reichert and Sussmann, 2016). However, all these observations typically cover only the mid-infrared. A few instruments have been developed to cover the far-infrared (FIR) region, defined as wavelengths greater than $15 \mu \mathrm{m}$ or, approximately, above the $\mathrm{CO}_{2}$ $v_{2}$ band, and are operated from ground and airborne platforms for limited timescale campaigns (Mariani et al., 2012; Green et al., 2012; Mlynczak et al., 2016).

While the relevance of the FIR spectral interval for atmospheric studies, and in particular for the study of climate, is a well-established concept (Sinha and Harries, 1995; Brindley and Harries, 1998; Harries et al., 2008), FIR still remains a significantly underexplored region, even more so if we consider specifically long-term monitoring projects.

The Radiation Explorer in the Far Infrared - Prototype for Applications and Development (REFIR-PAD) Fourier transform spectroradiometer (FTS) has been developed with the aim of performing spectrally resolved measurements of atmospheric emitted radiation covering most of the atmospheric emission spectrum, from 7 to $100 \mu \mathrm{m}$, thus including the FIR region.

The use of room-temperature detectors and of highly reliable mechanical solutions derived from space-qualified projects (Rizzi et al., 2002) makes the REFIR-PAD instrument an ideal tool to perform ground-based monitoring missions on climatologically relevant timescales. This capability was tested in 2007 with the ECOWAR (Earth COoling by WAter vapor Radiation) campaign (Bhawar et al., 2008) and in 2009 with the RHUBC-II campaign (Radiative Heating in the Underexplored Bands Campaign-II) (Turner et al., 2012). REFIR-PAD measurement capabilities are currently being fully exploited with the installation of the instrument at the Italian-French Antarctic station Concordia, in the Dome $\mathrm{C}$ region on the Antarctic Plateau $\left(75^{\circ} 06^{\prime} \mathrm{S}\right.$, $123^{\circ} 23^{\prime} \mathrm{E}, 3.233 \mathrm{~m}$ a.s.1.), where it has been operating in continuous acquisition mode since December 2011.

The REFIR-PAD Antarctic campaign is performed in the framework of several research programs financed by the Italian Antarctic Research Program (PNRA - Programma Nazionale di Ricerca in Antartide): PRANA (Proprietà Radiative del vapore Acqueo e delle Nubi in Antartide), COMPASS (COncordia Multi-Process Atmospheric StudieS), DOCTOR (DOme C Tropospheric ObserveR), and FIRCLOUDS (Far Infrared Radiative Closure Experiment For Antarctic Clouds).

Previous deployment of a FTS instrument at Dome C dates back to the austral summer season between 2003 and 2004, when the Polar AERI (P-AERI), operating in the 500$3000 \mathrm{~cm}^{-1}$ spectral range with $1 \mathrm{~cm}^{-1}$ resolution, was used to perform a characterization of the Antarctic DLR (Walden et al., 2005, 2006). Other similar measurements were performed at the South Pole (Town et al., 2005) and at Dome A (Shi et al., 2016).

In this paper a review of the main characteristics of the REFIR-PAD spectroradiometer is shown, together with the description of some measurement results obtained in groundbased campaigns in clear sky conditions; considerations and challenges related to the study of clouds are considered out of the scope of this work.

\section{The REFIR-PAD spectroradiometer}

The REFIR-PAD FTS is based on a Mach-Zehnder interferometer with a folded optical design that allows for a compact instrument while still retaining the moderate resolution and high throughput needed for atmospheric studies. The folding of the optical path and the number of reflections are designed to provide some degree of scanning mirror misalignment compensation (Carli et al., 1999a; Palchetti et al., 1999), allowing for a simpler mirror scanning mechanism design (Bianchini et al., 2006b).

The Mach-Zehnder configuration provides access to both of the two inputs and the two outputs of the interferometer, allowing for the use of a reference blackbody source (RBB in Fig. 1) permanently installed on the second input. This feature, as we will see later, is critical for the reduction of beam splitter emission effects. Moreover, output separation allows us to have two independent output channels.

The interferometer has the capability of operating in both a Martin-Puplett (Martin and Puplett, 1969) polarizing scheme and a more simple amplitude-division configuration. In the first case, as shown in Fig. 1a, all four beam splitters are installed, two acting as a polarization divider and recombiner and the other two, the ones nearer to the mirror scanning mechanism (rooftop mirror unit, RTMU in figure), as proper interferometric beam splitters.

The amplitude-division configuration makes use of only the two interferometric beam splitters, while the two other mounts are left empty. This configuration has been shown to be the best choice when aiming for a wide operating spectral range since with the use of bilayer dielectric beam splitters the instrumental response can be tuned according to the experimental requirements.

For example, with a $0.85 \mu \mathrm{m}$ Ge layer on a $2 \mu \mathrm{m}$ Mylar substrate, an interferometric efficiency of better than $80 \%$ in the $100-1300 \mathrm{~cm}^{-1}$ spectral range can be achieved, while with a thinner structure $(0.6 \mu \mathrm{m}$ Ge layer on a $1.5 \mu \mathrm{m}$ Mylar substrate) the response towards higher wavenumbers can be enhanced, extending the operating range to $1900 \mathrm{~cm}^{-1}$ at the cost of a reduction of the efficiency below $200 \mathrm{~cm}^{-1}$ (see Fig. 2). This does not constitute a problem for ground-based measurements in which even in cases of extreme atmospheric 


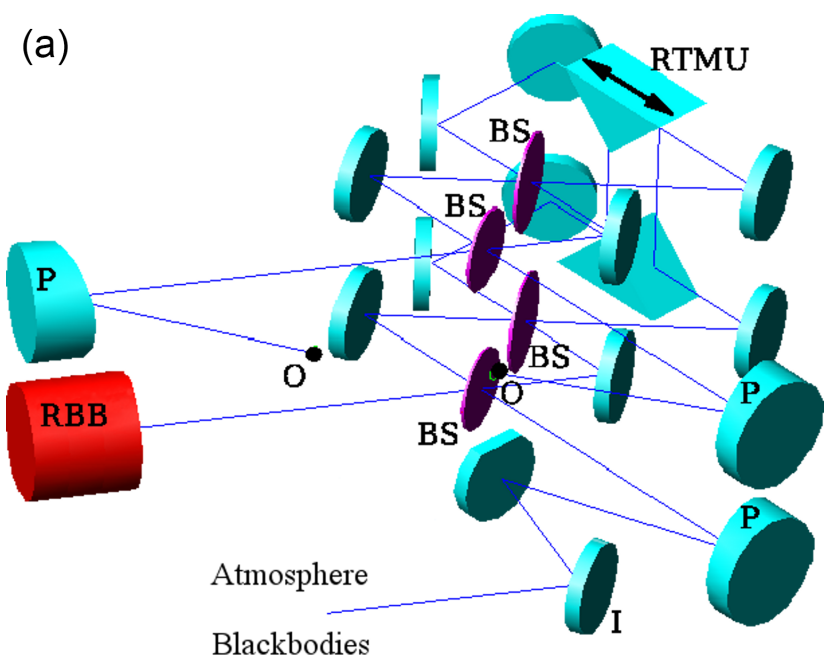

(b)

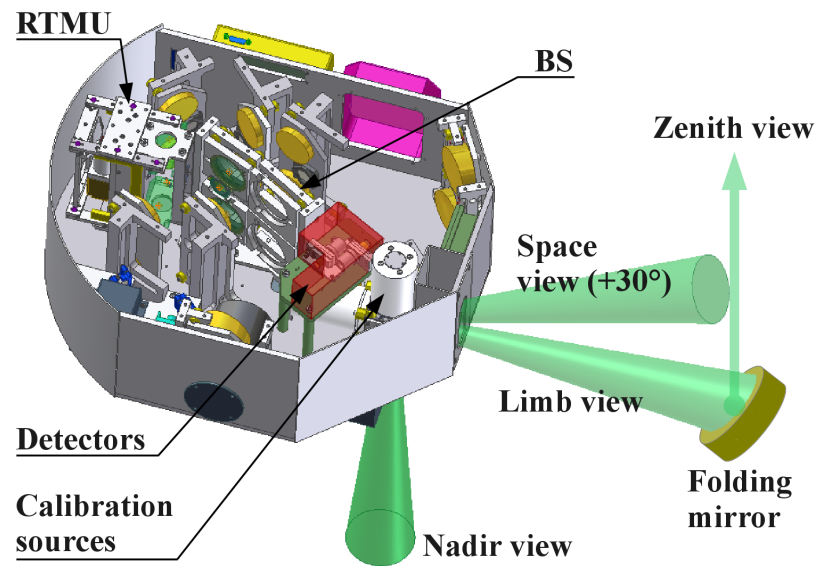

Figure 1. (a) REFIR-PAD optical layout. BS: beam splitters; P: offaxis parabolic mirrors; RBB: reference blackbody source; O: outputs (detectors); I: input selection mirror; RTMU: rooftop mirror unit (interferometric scanning mirror). (b) REFIR-PAD mechanical layout showing the actual placement of the components in the instrument enclosure and the optional folding mirror used for zenith view.

transparency, with very low humidity, there is no significant atmospheric signal below $200-250 \mathrm{~cm}^{-1}$.

In Fig. 2 it is also evident that the substrate itself poses some limitations to the operating spectral range due to its absorption properties. The substrate absorption bands not only reduce the efficiency, possibly "blinding" the instrumental response as in the case of the strong features near 1250 and $1700 \mathrm{~cm}^{-1}$, but also introduce a de-phasing that makes an accurate radiometric calibration a challenging task in spectral regions close to the absorption bands (Bianchini and Palchetti, 2008).

These problems could be overcome by using a different substrate, like polypropylene, which has fewer and weaker absorption bands in the region of interest, but this comes at

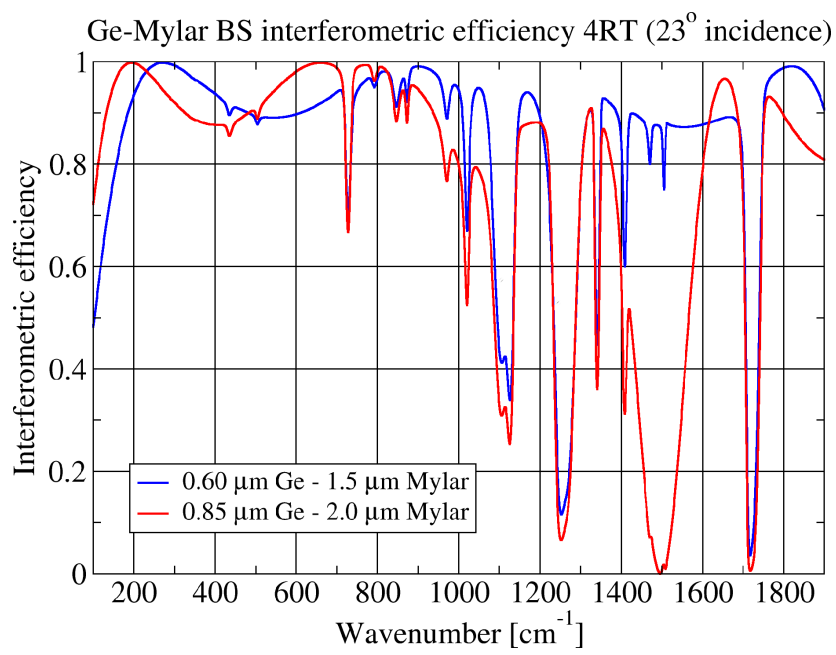

Figure 2. Real part of the interferometric efficiency (calculated as 4 times the product of reflectivity $R$ and transmissivity $T$ ) for two different configurations of the bilayer germanium on Mylar substrate beam splitters.

the cost of worse optical and mechanical properties, which can critically affect the delicate process of beam splitter assembly and germanium deposition. So Mylar has been chosen as a trade-off between theoretical efficiency and optical quality.

Problems arising from beam splitter substrate absorption, and in general from nonideal beam splitters, are also mitigated through design choices in the interferometer: the use of a reference source (RBB in Fig. 1) operating at the same temperature of the instrument, and thus of the beam splitters, ideally reduces the contribution to the interferogram due to beam splitter emission to zero (Carli et al., 1999b; Bianchini et al., 2009). The orientation of the two beam splitters is also chosen in order to symmetrize the optical paths and minimize the out-of-phase contributions to the interferogram (Bianchini et al., 2009), as will be discussed in more detail in Sect. 8. As a matter of fact, the biggest contribution to the interferometer output due to the beam splitters in this configuration comes from the small layer thickness differences between the two beam splitters, differences that are inherent in the manufacturing process (see also Sect. 8).

A rotating folding mirror is placed at the instrument input port, allowing us to select an atmospheric line of sight or one of the two onboard calibration sources. The rotating mirror is in the focus of a $320 \mathrm{~mm}$ focal length, $20^{\circ}$ off-axis parabolic mirror that collimates input radiation towards the interferometer. The second input does not need collimating optics since it is directed towards the large-diameter RBB source.

The zenith line of sight that is used in the case of groundbased measurements is obtained through the use of an extra folding mirror placed on the limb line of sight (see Fig. 1b).

Two $170 \mathrm{~mm}$ focal length, $30^{\circ}$ off-axis parabolic mirrors focus the interferometer output ports on two $10 \mathrm{~mm}$ diameter 


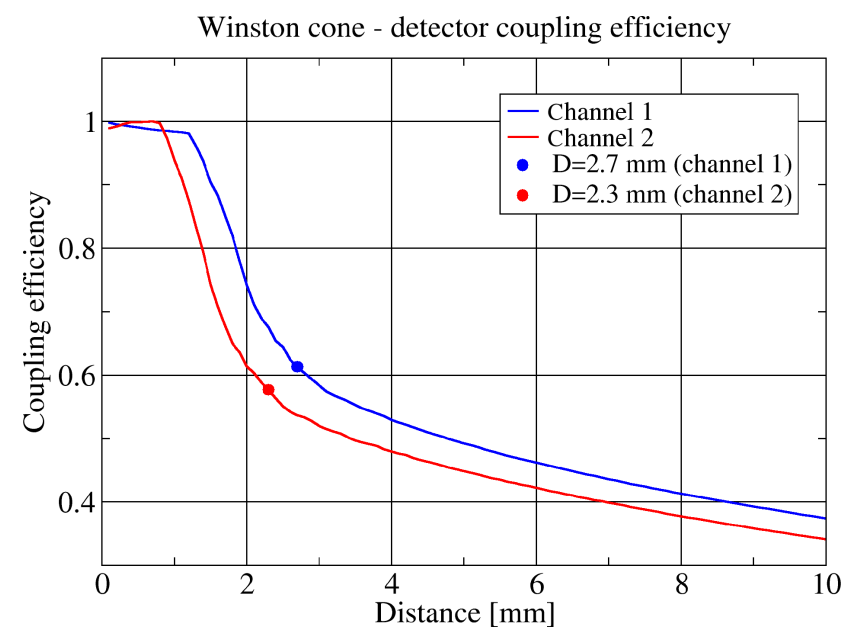

Figure 3. Plot of the concentrator-detector coupling efficiency as a function of their distance for both channels. The solid dots show the actual operating point, corresponding to a concentrator-detector distance of $2.7 \mathrm{~mm}$ for channel 1 and $2.3 \mathrm{~mm}$ for channel 2 .

Winston cone concentrators that feed the detectors. The interferometer is placed in the $1.4 \mathrm{~m}$ length collimated optical path between input and output parabolic mirrors. A $22 \mathrm{~mm}$ pupil stop is placed in the center of the collimated path, inside the rooftop mirror unit.

The designed beam divergence $\Omega$ inside of the interferometer is $0.0027 \mathrm{sr}$, giving an instrument throughput of $0.011 \mathrm{~cm}^{2} \mathrm{sr}$. However, in practice there is a limitation that is posed by the concentrator-detector coupling.

The coupling efficiency is limited by the presence of a CsI window that seals the detector case from ambient humidity. Ideally the detector should be placed as near as possible to the concentrator output aperture, but the minimum distance is actually limited by the window thickness and the distance between the window and the detector active surface.

In Fig. 3 the variation in the coupling efficiency with the distance between concentrator and detector is shown. The curves corresponding to the two channels differ due to the diameter of the active surface of the two detectors $(2 \mathrm{~mm}$ for channel 1 and $1.5 \mathrm{~mm}$ for channel 2). The dots show the operating condition of the two channels, corresponding to a concentrator-detector distance of $2.7 \mathrm{~mm}$ for channel 1 and $2.3 \mathrm{~mm}$ for channel 2 .

As shown in Fig. 3, the limitation in coupling efficiency causes a loss of about $40 \%$ in signal but also acts as a field stop limiting the instrument field of view, reducing the beam divergence to about $0.00087 \mathrm{sr}$, for a throughput of about $0.0035 \mathrm{~cm}^{2} \mathrm{sr}$.

All the mirrors used on the REFIR-PAD instrument are coated in bare gold in order to minimize infrared absorption. Since the zenith-looking folding mirror is placed outside the calibration path, its reflectivity has been characterized in the laboratory and its temperature is constantly monitored in or- der to apply a calibration adjustment. The effect of polarization is estimated to be negligible, taking into account the fact that the instrument is not operating in polarization mode and the zenith scene, in clear sky conditions, is not polarized.

Interferometric metrology is based on a paraxial laser interferometer with a $780 \mathrm{~nm}$ laser source (Bianchini et al., 2000) that has been thoroughly tested in high-resolution FTS instruments operating from both the ground (Palchetti et al., 2005) and stratospheric platforms (Bianchini et al., 2004, 2006a).

The reference interferometer does not share any of the infrared interferometer optics, simplifying the instrument design and alignment, at the cost of having a possible misalignment between the two optical axes. This does not constitute a problem since it induces a linear wavenumber error that is taken care of in the wavenumber calibration procedure. This procedure, further detailed in Sect. 7, is based on known atmospheric line centers and does not rely on the measurement of the exact laser wavelength.

Along with the RBB, two other blackbody sources are used for the radiometric calibration (Bianchini and Palchetti, 2008). These sources, hot blackbody (HBB) and cold blackbody $(\mathrm{CBB})$, are placed near the instrument measurement port and can be switched into the line of sight through the rotating input bare gold mirror (see Fig. 1, label "Calibration sources").

For the HBB and CBB the emissivity is better than 0.999 and the operating temperature is between 10 and $80^{\circ} \mathrm{C}$. Temperature stability and temperature measurement uncertainty are both about $0.3 \mathrm{~K}$, while gradients are within $0.5 \mathrm{~K}$ (Palchetti et al., 2008).

It should be noted that limited size and good emissivity can both be achieved with these sources due to the relatively small $22 \mathrm{~mm}$ aperture of the blackbody, which is a consequence of the placement in proximity of the focus of the input parabolic mirror.

The RBB has a larger diameter $(64 \mathrm{~mm})$ due to its placement in a collimated part of the optical path, but its requirements are more relaxed since it is not stabilized in temperature but, instead, left in thermal equilibrium with the instrument.

Acquisition of HBB and CBB radiance is performed regularly in order to obtain a constant tracking of possible instrumental response function variations. Typically, a $10 \mathrm{~min}$ acquisition sequence includes four atmospheric measurements and four calibrations, two with the HBB and two with the CBB. Radiometric performances of the REFIR-PAD instruments are further described in Sect. 6 .

\section{Instrumental line shape}

A good model of the instrumental line shape (ILS) is a necessary requirement to correctly interpret the measured spectra and perform the level 2 data analysis (Sect. 10). Several ef- 
fects can contribute to distort the ILS from the theoretical $\operatorname{sinc}\left(2 \pi \sigma z_{\max }\right)$ function, where $z_{\max }$ is the maximum optical path difference. Misalignment of the interferometer and scanning mirror deviations (Bianchini and Raspollini, 2000) can contribute to the ILS; another possible effect is due to the finite solid angle $\Omega$ of the radiation propagating inside of the interferometer.

The effect of the finite solid angle is to broaden and shift spectral lines by convolving, in the wavenumber domain, the ideal sinc ILS with a box function extending from 0 to $\sigma_{0} \Omega / 2 \pi$, where $\sigma_{0}$ is the spectral line center (Vanasse and Sakai, 1967).

Thus, in the optical path difference domain, the effect gives an additional, wavenumber-dependent, apodization term $\operatorname{sinc}\left(z \sigma_{0} \Omega / 2\right)$ to be multiplied by the standard boxcar function extending from $-z_{\max }$ to $z_{\max }$. The dependency on wavenumber of the apodization function makes the exact treatment of such an effect a difficult task in the case of a broadband spectrum. A possibility is to consider $\sigma_{0}$ a constant, equal to the central wavenumber of the operating spectral band.

Moreover, if $\pi / \sigma_{0} \Omega \gg z_{\max }$ the solid angle contribution to the ILS is small and can be approximated with a triangular component in the apodization. The resulting apodization function can thus be treated as a linear combination of a boxcar and a triangle function with $\alpha$ and $1-\alpha$ coefficients, where $\alpha=\operatorname{sinc}\left(z_{\max } \sigma_{0} \Omega / 2\right)$.

This is a rough approximation with respect to the exact mathematical treatment of the ILS function, but since in normal instrumental operating conditions the deviations from the "ideal" ILS are very small, the effect of the approximation is negligible, and the calculation of the ILS is much faster since it makes use of the two simplest apodization functions.

REFIR-PAD ILS has been analyzed through HBB calibration measurements in which isolated water vapor lines coming from residual humidity in the instrument have been identified. These features are weak enough to be far from saturation and have a natural linewidth negligible with respect to ILS. In Fig. 4 the results for two different lines are shown, one in the FIR region, at $526 \mathrm{~cm}^{-1}$, and one at the edge of the REFIR-PAD operating region, at $1430 \mathrm{~cm}^{-1}$. The top panels in Fig. 4 (red lines) correspond to acquisitions performed with a $0.25 \mathrm{~cm}^{-1}$ spectral sampling, and the bottom panels (blue lines) to a $0.5 \mathrm{~cm}^{-1}$ spectral sampling.

The measured lines are fitted with the $\alpha \cdot \operatorname{sinc}+(1-\alpha) \cdot \operatorname{sinc}^{2}$ approximated line shape, corresponding to the combination of a boxcar and a triangular apodization, obtaining the corresponding value for $\alpha$.

The $\alpha$ coefficient has also been retrieved, as a function of wavenumber, for several other different spectral lines. The result of this kind of analysis is shown in Fig. 5, where average $\alpha$ values are plotted vs. wavenumber. Two different series of measurements were analyzed: some performed with $0.25 \mathrm{~cm}^{-1}$ (red circles) and others with $0.5 \mathrm{~cm}^{-1}$ (blue

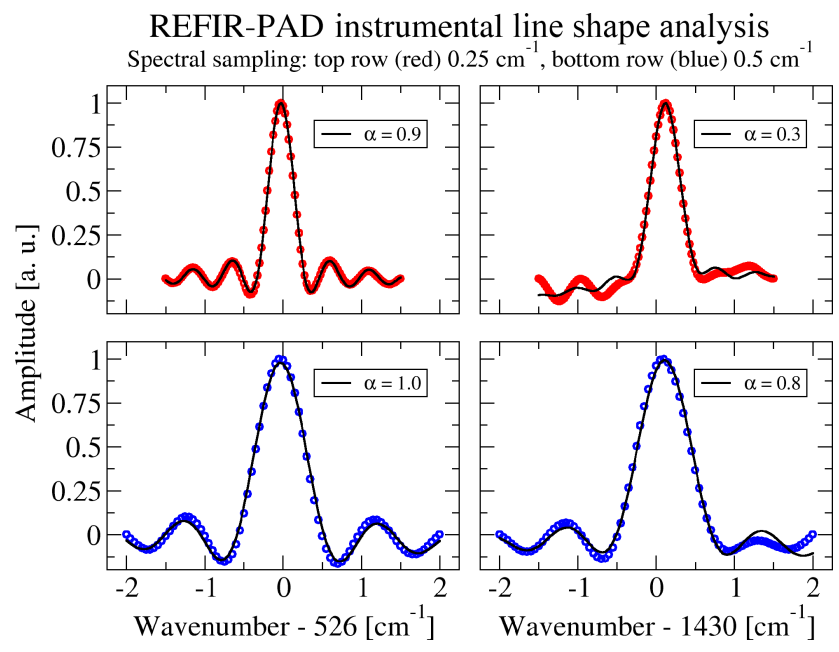

Figure 4. REFIR-PAD instrumental line shape. The blue line shows the isolated atmospheric line used for the analysis; the instrumental line shape is a linear combination of the sinc and $\operatorname{sinc}^{2}$ components.

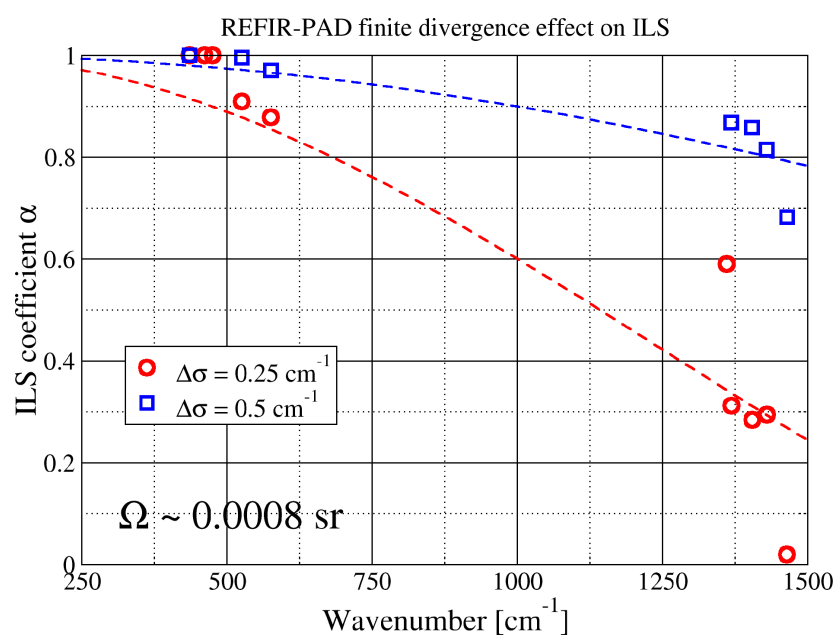

Figure 5. REFIR-PAD instrumental line shape coefficient obtained through analysis of measured spectra for 0.25 and $0.5 \mathrm{~cm}^{-1}$ nominal resolution. Continuous lines show the $\operatorname{sinc}\left(z_{\max } \sigma_{0} \Omega / 2\right)$ theoretical behavior.

squares) spectral sampling. The theoretical expression $\alpha=$ $\operatorname{sinc}\left(z_{\max } \sigma \Omega / 2\right)$ is also plotted, with the $\Omega$ value fitted to the experimental data.

Both the datasets provide the same $\Omega$ value, as expected. The fitted value $(0.0008 \mathrm{sr})$ is also smaller than the theoretical beam divergence given by the optical design (about $0.0027 \mathrm{sr}$ ), but in good agreement with the actual value of $0.00087 \mathrm{sr}$ calculated taking into account the limitations in coupling efficiency due to the finite distance between Winston cone output aperture and detectors (see Sect. 2).

It should be noted that at low wavenumbers the solid angle contribution is completely negligible, but even in this case the line fitting gives a $\alpha$ value of lower than 1 (typically about 
0.95). This can be explained with the fact that there are other contributions to the ILS (residual misalignment, optics planarity, scanning mirror movement irregularities) that can give a residual contribution that is visible when the solid angle effect is negligible.

We also observe that, since in case of a small amount of interferometric misalignment the effect on the ILS can be approximated at the first order with an increase in the $\operatorname{sinc}^{2}$ component, it is possible to treat the interferometric misalignment in level 2 data analysis through fitting the $\operatorname{sinc} / \operatorname{sinc}^{2}$ ratio as an extra parameter (see Sect. 10). This is a very useful feature in the case of remote operation in extreme environments, an operating condition in which a slight misalignment is always a possibility.

\section{Detectors and data acquisition electronics}

One of the defining characteristics of the REFIR-PAD spectroradiometer is the use of room-temperature detectors to cover the middle to FIR spectral range. This result is obtained through the use of high-sensitivity deuterated Lalanine-doped triglycine sulfate pyroelectric detectors provided by BAE-Selex (models P5315 and P5541). Specifications for the P5315 (P5541) at $f=100 \mathrm{~Hz}$ are detectivity $D^{*}=5.0 \times 10^{8}\left(5.3 \times 10^{8}\right) \mathrm{cm} \mathrm{Hz}^{1 / 2} \mathrm{~W}^{-1}$ and responsivity $1250(450) \mathrm{V} / \mathrm{W}$.

The detector active area diameter is $2 \mathrm{~mm}$ for P5315 and $1.5 \mathrm{~mm}$ for P5541. To enhance the light-gathering ability of the detectors, Winston cone concentrators are mounted in front of them (see Sect. 2).

The detectors are specified for a $10-3000 \mathrm{~Hz}$ operating frequency range. In standard operating conditions $(3.3 \times$ $10^{-2} \mathrm{~cm} \mathrm{~s}^{-1}$ optical path difference scanning speed, 100 $1500 \mathrm{~cm}^{-1}$ spectral range) the REFIR-PAD instrument operates in the $3.3-49.5 \mathrm{~Hz}$ frequency interval. This is partially outside of the low end of the specified operating range; thus an accurate characterization of the detector system is required.

The typical frequency response of a pyroelectric detector is characterized by "crossed" low and high cutoffs resulting in a strongly frequency-dependent amplitude and phase. The presence of a low-frequency cutoff is rather an advantage in an intrinsically AC-coupled application like FT spectroscopy, but, conversely, a frequency-dependent de-phasing constitutes a severe problem in a FT spectrometer and must be solved by the use of a specifically designed preamplifier with a tailored response function in order to obtain a flat response and a very low de-phasing across the operating frequency range.

In Fig. 6 the response of the detector and preamplifier is measured in operating conditions, supplying the detector with an optical step function through the use of a laser and a shutter. The resulting response function can be fitted with a mathematical model of the detectors' two-pole response mul-
REFIR-PAD detector \& preamplifier response function - 01/2016

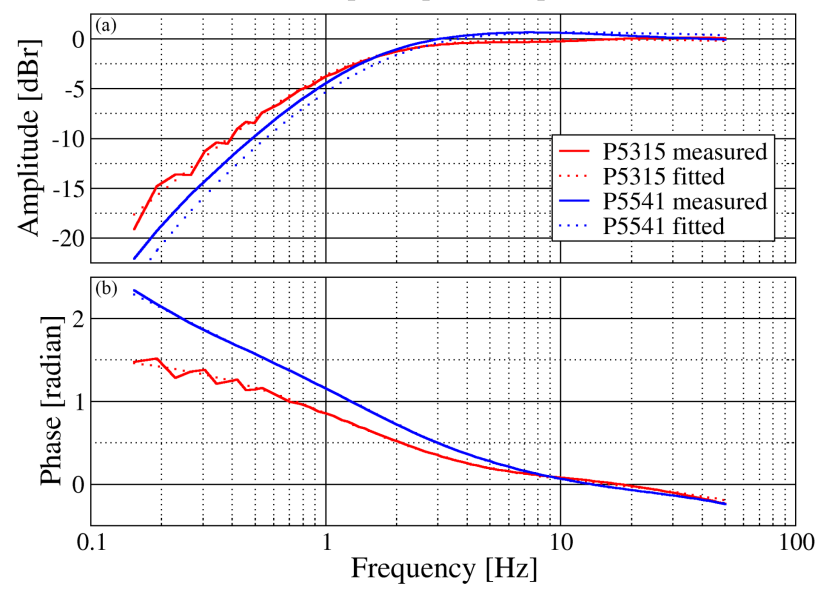

Figure 6. REFIR-PAD detector preamplifier response as measured in normal operating conditions, along with a fit of the theoretical model used to measure the detectors' characteristic low- and highpass frequencies.

tiplied by the preamplifier electronics response in order to obtain an estimate of the actual frequencies of the detector poles. The fitted function is shown in Fig. 6 as a dotted line and is in very good agreement with the measured data.

The values for the low- and high-frequency cutoffs obtained by the fitting process shown in Fig. 6 are used, together with the mathematical model of the preamplifier response, to provide an estimate of the residual de-phasing to be used in the phase correction algorithm in the level 1 processing of the interferograms (Bianchini and Palchetti, 2008).

\section{Control and data storage system}

The REFIR-PAD instrument features an onboard control unit that allows for autonomous operation (shown as a purple box in the mechanical layout shown in Fig. 1).

The onboard control unit is based on a PC-104 industrial computer with a $486 \mathrm{DX} 2 \mathrm{CPU}$ operating at $100 \mathrm{MHz}$ and $32 \mathrm{MB}$ of RAM. Storage is provided by a $64 \mathrm{~GB}$ SSD or, alternatively, a removable CompactFlash card slot (the use of which bypasses the SSD).

The onboard unit runs a streamlined version of Debian GNU/Linux v. 3.0 in which all the nonessential services have been disabled to reduce system disk access and thus increase robustness in the case of loss of power. At system boot, after enabling networking, the REFIR-PAD control program is launched, immediately starting the data acquisition sequence.

This setup allows for compactness and robustness and is ideal for the balloon-borne operation mode and for short ground-based campaigns: the SSD can store up to a month of continuous measurements, while the CompactFlash slot allows us to easily retrieve data after a measurement run. 
The permanent installation at Concordia station has instead required an upgrade of the control systems surrounding the REFIR-PAD instrument in order to provide continuous, unattended operation capabilities.

At Concordia station the REFIR-PAD instrument is installed indoors, in a shelter near the main base, enclosed in a thermally insulated box that is connected to an opening on the shelter roof by means of an insulated chimney. In this way, even if no window is used to separate the instrument from the outside environment, the shelter inside is kept protected from the outside air. The measurement port on the roof of the shelter can be closed by means of a motorized door when the instrument is not operated (and closes automatically in the case of loss of power).

An autonomous microcontroller-based thermal control system is used to keep the instrument at a constant temperature (within $\pm 0.5 \mathrm{~K}$ ) through a set of heaters and a fan-driven inlet tube extracting cool air from the bottom of the shelter. The thermal control system is also provided an Ethernet connection and can be remotely controlled and configured through a minimal web interface.

The REFIR-PAD FTS is remotely operated through a second computer placed in the shelter and connected with a direct point-to-point Ethernet link to the FTS onboard control unit. This control and storage computer can switch on power to the FTS, to the view port door, and to the heating system. It does also share, through the Network File System (NFS) protocol, a 2 TB RAID-1 disk array, which is mounted at boot by the REFIR-PAD onboard control unit in order to store the acquired data.

The normal operation sequence of this setup consists in the control and storage computer opening the measurement port, turning on REFIR-PAD, and waiting for a preset interval (typically configured as about 5-6h).

At the end of the measurement run, it shuts down REFIRPAD through the network and proceeds to compress and archive the raw data in a time-stamped directory structure and finally to perform level 1 preprocessing.

This pre-processing step is needed in order to send the calibrated and averaged DLR spectra to Italy since the full number of raw data produced by the FTS is too large for a direct transfer.

After the end of the data preprocessing, a new measurement run is started.

Together with REFIR-PAD data, the control and storage computer performs acquisition and storage of several auxiliary parameters ranging from weather parameters outside the shelter measured by a Vaisala WXT520 station to diagnostic temperature values coming from different sensors placed in the shelter and inside of the instrument box.

The control and storage computer is always on and can be accessed remotely, allowing for the complete control of all the acquisition parameters even when REFIR-PAD is not operating since the configuration files reside on the RAID-1 disk array.
Advantages of this architecture are redundancy and failsafe operation: in the case of a malfunction of the control and storage unit the REFIR-PAD instrument can still operate autonomously within the 1-month data storage autonomy provided by the onboard SSD. The control and storage unit can be easily replaced with a pre-configured, identical spare unit available in the shelter. Conversely, in the case of a malfunction of the REFIR-PAD onboard unit, a serial console active at boot time and accessible from the control and storage unit allows for remote troubleshooting including poweron self-test monitoring and BIOS configuration.

The raw data number produced by the REFIR-PAD instrument is about $40 \mathrm{~GB}$ per day (2 GB per day compressed); the corresponding level 1 preprocessing output is about $50 \mathrm{MB}$ per day (12 MB per day compressed), a number of data that can be easily transferred even with the low-bandwidth connection provided at Concordia station $\left(512 \mathrm{~kb} \mathrm{~s}^{-1}\right.$ maximum).

\section{Radiometric performances}

A direct estimate of the radiometric accuracy of the REFIRPAD spectra can be obtained through the signal measured in a spectral interval in which complete atmospheric transparency is expected. In the case of a high-altitude, extremely dry environment, this condition is achieved in the atmospheric window of around $800-1000 \mathrm{~cm}^{-1}$.

Specifically we used the dataset acquired from the Cerro Toco site at about $5500 \mathrm{~m}$ a.s.1. in the Atacama region, Chile, during the RHUBC-II campaign (Turner et al., 2012). Of this dataset, we selected only measurements that have a precipitable water vapor (PWV) of lower than $0.6 \mathrm{~mm}$. In these cases the expected atmospheric radiance signal in a narrow interval between 828 and $839 \mathrm{~cm}^{-1}$ is completely negligible, so this spectral region can be effectively used to check the instrument radiometric accuracy in the middle of its operating band.

In Fig. 7 a statistical analysis of the distribution of the average radiance in the selected interval is presented. The distribution has been fitted with a Gaussian curve, obtaining a negligible offset and a standard deviation of about $0.7 \mathrm{~mW}\left(\mathrm{~m}^{2} \mathrm{srcm}^{-1}\right)^{-1}$. The latter is in a good agreement with the a priori estimate of the radiometric error obtained combining the noise equivalent spectral radiance (NESR) and the calibration error (Bianchini and Palchetti, 2008): the estimated NESR in the selected spectral band is $<0.6 \mathrm{~mW}\left(\mathrm{~m}^{2} \mathrm{srcm}^{-1}\right)^{-1}$ and the calibration uncertainty is $0.6 \mathrm{~mW}\left(\mathrm{~m}^{2} \mathrm{srcm}^{-1}\right)^{-1}$, giving a total uncertainty (through root sum of squares) of about $0.85 \mathrm{~mW}\left(\mathrm{~m}^{2} \mathrm{srcm}^{-1}\right)^{-1}$.

The much lower value of constant bias shows that systematic errors in the calibration procedure are negligible with respect to the estimated radiometric uncertainty.

This method for checking the calibration accuracy does not provide a characterization through the whole spectral 


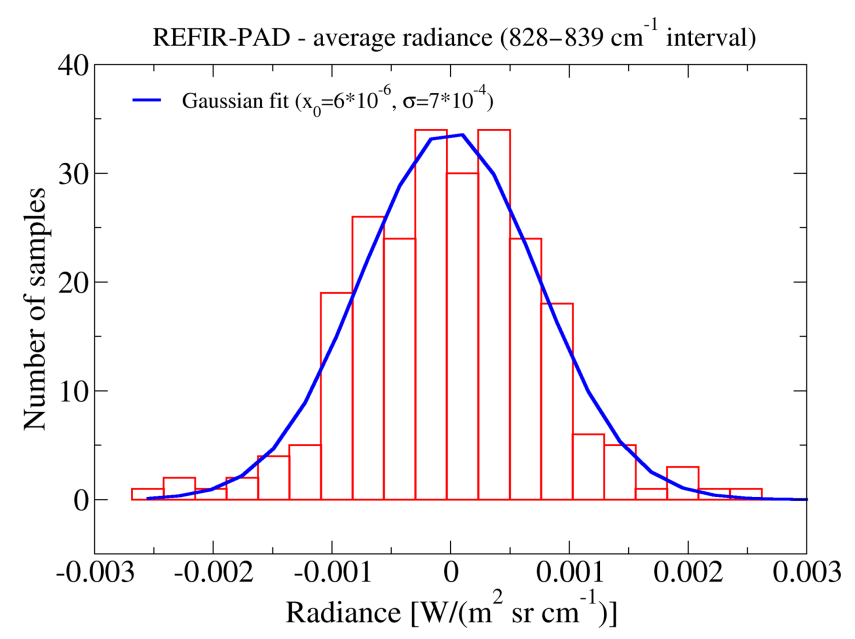

Figure 7. Statistical analysis of the average radiometric signal in a high-transparency window $\left(828-839 \mathrm{~cm}^{-1}\right)$ for RHUBC-II measurements. Radiometric bias is close to zero, while the half width of the Gaussian distribution is $0.7 \mathrm{~mW}\left(\mathrm{~m}^{2} \mathrm{srcm}^{-1}\right)^{-1}$.

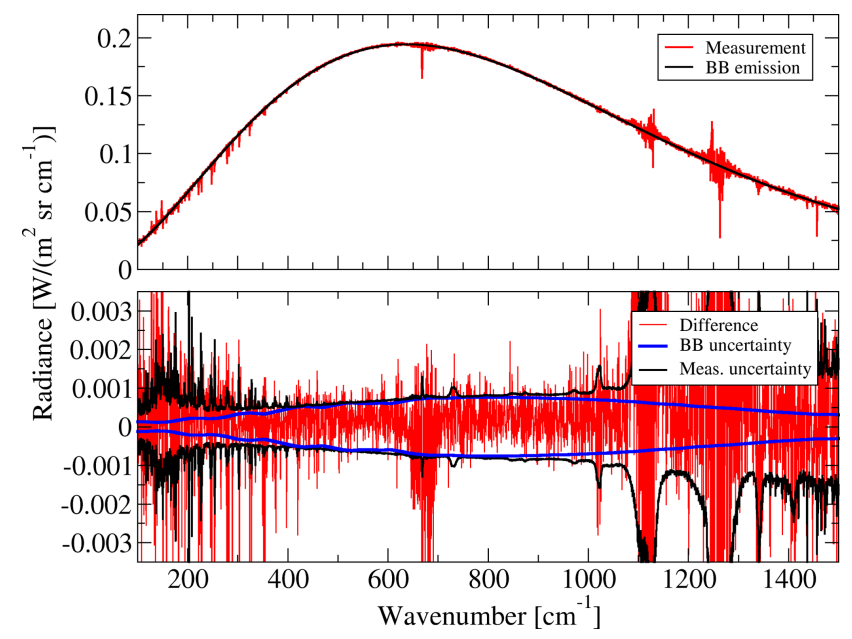

Figure 8. Measurement of a reference calibration blackbody source at about $324 \mathrm{~K}$.

range, but can be performed whenever needed, during a multiyear deployment of the instrument, provided very dry atmospheric conditions are present (which is frequently the case for Dome C). An estimate of the calibration error covering the full spectral range can instead be obtained only with a dedicated measurement performed using an external RBB placed on the instrument measurement port. Figure 8 shows the results of such a calibration measurement (red line in the top panel) compared with the RBB calculated emission (black line in the top panel). In the bottom panel, the difference (gray line) is compared with the estimated measurement uncertainty (black line) and the blackbody calculated emission uncertainty (blue line).

The results show that the calibration accuracy is quite constant over the $300-1000 \mathrm{~cm}^{-1}$ spectral range. Below
$300 \mathrm{~cm}^{-1}$, and in correspondence with the beam splitter substrate absorption bands above $1000 \mathrm{~cm}^{-1}$, the measurement errors are prevalent and it is difficult to quantify the actual calibration accuracy.

\section{Spectral calibration}

The use of a diode laser as a metrology source for the REFIRPAD spectroradiometer has allowed for increased ruggedness and compactness of the system; however, this is at the cost of a lower absolute stability of the spectral calibration reference.

The wavelength of a diode laser emission depends strongly on both the diode temperature and drive current. The unit used in REFIR-PAD features a specifically designed control unit providing both temperature stabilization and a highstability, low-noise current drive. In addition to temperature and drive current, diode lasers typically feature a large device-to-device wavelength variability. For this reason, once the reference source is installed and set up, its wavelength is calibrated and stored as a level 1 analysis software configuration parameter.

The main source of laser frequency error is due to thermal drifts in the temperature and current control and has been evaluated from the electronic component specifications in contributions of about $120 \mathrm{MHz} \mathrm{K}^{-1}\left(0.31 \mathrm{ppm} \mathrm{K}^{-1}\right)$ from laser current and $60 \mathrm{MHzK}^{-1}\left(0.16 \mathrm{ppm} \mathrm{K}^{-1}\right)$ from laser temperature.

Assuming a maximum temperature fluctuation of $2 \mathrm{~K}$, a safe estimate considering the performances of the instrument temperature control subsystem (see Sect. 5), we obtain a laser frequency error of better than $1 \mathrm{ppm}$. This, in normal operation, allows for the use of a single frequency calibration even in the case of long-term measurements.

The observed laser frequency drift (see Sect. 11) is about $<15$ ppm year $^{-1}$, still low enough to allow us to perform frequency calibrations monthly or even yearly. Nevertheless, a more robust automatic frequency calibration procedure has been developed to treat specific cases in which the abovementioned frequency stability cannot be reached, e.g., in the case of laser mode jumps, or large temperature drifts of the instrument environment.

The procedure is based on the line fitting of the residual absorption due to the $\mathrm{CO}_{2} v_{2}$ band that is observed in the HBB calibration measurements. This approach has been chosen in order to have a reference spectrum that is as independent as possible from the measurement conditions, so the calibration procedure does not need to be adjusted according to the observed scene. Also, the absorption spectrum used in the procedure can be simply modeled using line strengths and the sinc $+\operatorname{sinc}^{2}$ instrumental line shape (see Sect. 3). The downside of this approach is that a per-spectrum calibration cannot be performed since a calibration coefficient is obtained only from the HBB measurements. This does not constitute a limitation as long as the laser frequency drifts are negligible on 


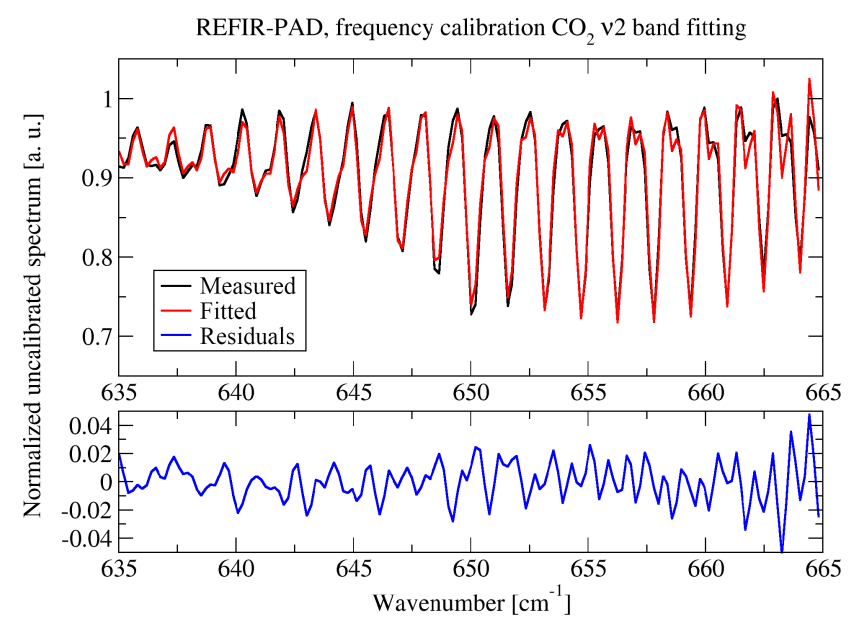

Figure 9. Result of the line fitting process used to perform automatic frequency calibration of the REFIR-PAD measured spectra.

the timescale of the calibration measurement repetition rate (about $10 \mathrm{~min}$ ). In Fig. 9 a sample result of the fitting process is shown.

Since the frequency fluctuation that a diode laser can experience can be quite large, a two-step fitting procedure has been developed. First a simple peak-finding algorithm is applied to the $\mathrm{Q}$ branch of the $\nu_{2}$ band, and then the fitting of the $\mathrm{P}$ branch in the $635-665 \mathrm{~cm}^{-1}$ spectral region is performed, as shown in Fig. 9. The first stage of the process prevents, in case of a frequency drift that is larger than the $\mathrm{P}$ branch line spacing, a systematic error in the second stage due to the periodic structure of the spectrum.

The last step needed to perform the frequency calibration of the atmospheric measurements involves a linear regression in time of the frequency shift coefficients obtained from the HBB measurements, the result of which is used to calculate the frequency drift correction for each of the atmospheric measurements.

In Fig. 10 the effect of the automatic frequency calibration procedure in two different case studies is shown. In both cases the frequency shift retrieved by the level 2 data analysis (see Sect. 10) is plotted with standard (blue curve) and automatic (red curve) frequency calibration.

During December 2014 (top panel in Fig. 10) the reference laser showed bistable operation due to operating parameters being near a mode jump. This caused the laser to operate on randomly one or the other of the two nearby modes. The separation between modes corresponds to a $400 \mathrm{ppm}$ frequency shift (blue curve). After automatic frequency calibration it can be seen that the frequency shift coefficient variability (red curve) is reduced to the effect of measurement noise on level 2 analysis.

In the bottom panel in Fig. 10 the same method applied to a dataset from December 2017, at the end of the 2-year period shown in Fig. 18, is shown. While the overall effect observed

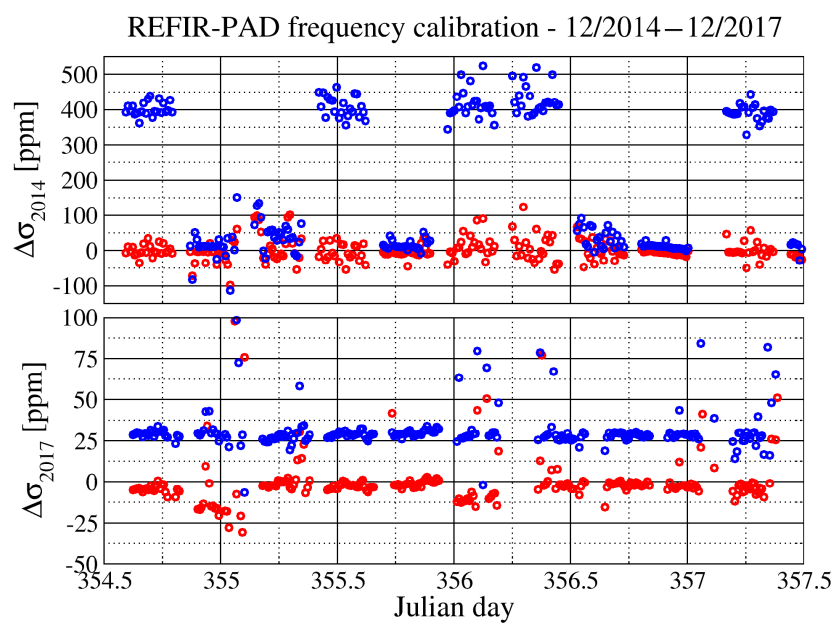

Figure 10. Effect of the automatic frequency calibration procedure. Blue circles represent the frequency correction factor retrieved by the level 2 data analysis process with uncorrected data; red circles show the same parameter retrieved from automatically frequencycalibrated spectra.

is a general reduction of the $25 \mathrm{ppm}$ offset, it should be noted that the automatic calibration procedure itself induces fluctuations that can be as large as $10 \mathrm{ppm}$ due to the accuracy of the fitting process.

\section{Instrument mathematical modeling}

A simulation software has been developed with the intent of providing a tool to estimate the expected performance of the REFIR-PAD interferometer. The code is written in MATLAB-compatible language and takes into account all relevant elements of the instrument geometry and optical design. The main scope of this tool is to assist in the design and test of the beam splitters and to identify and verify the configuration providing the best optical path compensation.

The simulation assumes a generic Mach-Zehnder design with two independent inputs and two outputs. The beam splitters are modeled as an asymmetric multilayer structure characterized by generally different optical reflectivities for the two sides $R_{1}$ and $R_{2}$.

In the simulation the two inputs can be associated with a blackbody source or with a synthetic spectrum provided by an atmospheric forward model, so in both cases a real function. The two sources are split according to the calculated complex transmission and reflection coefficients of the first beam splitter as obtained by the dielectric multilayer theory using the measured complex refraction indexes for the different layers in order to correctly represent bulk material absorption.

The emission of the first beam splitter, due to its non-null absorption, is also considered to be an independent source. Beam splitter absorption is mainly caused by the substrate 
absorption bands, so it appears as a localized and easily identifiable effect.

The two arms of the interferometer are then recombined on the second beam splitter whose properties are calculated in the same way as for the first.

Effect of the misalignment of interferometric components is calculated in the circular beam approximation using Bessel functions; planarity error is also modeled using simple approximations (spherical or trapezoidal deformation) and integrated into the beam profile.

The resulting complex spectrum is multiplied for a real absorption spectrum simulating the effect of air inside of the instrument (most of the absorption takes place outside of the interferometric path; thus it does not produce de-phasing) and the effect of the detector windows, obtaining the total signal incoming on both detectors. An inverse FT is applied to this signal to generate the simulated interferogram, which is then processed with the standard level 1 data analysis chain used to process the REFIR-PAD measurements and described in detail in Bianchini and Palchetti (2008).

In Fig. 11 a comparison between measurements and simulation of an acquisition of the internal HBB source is shown. Figure 11a shows amplitude and phase of the signals observed on the two output channels in the case of a setup in which the beam splitter coated surfaces are facing opposite directions. Red lines correspond to measured spectra and blue lines to simulations. Figure $11 \mathrm{~b}$ shows the same signals obtained in a configuration in which the beam splitter coated surfaces are facing the same way; i.e., the configuration occurring in the case of a single homogeneous beam splitter surface is used as both a beam divider and recombiner in the Mach-Zehnder interferometer. The tests were performed with the $0.85 \mu \mathrm{m} \mathrm{Ge}$ on $2.0 \mu \mathrm{m}$ Mylar beam splitter design.

It appears evident that the second configuration does not allow for a good compensation of the optical paths, as can be seen by the large oscillations in the phases. This improves greatly with the use of opposite-facing beam splitters. It should be noted that, as the model confirms, the residual phase undulations that are still observed in the latter configuration come from small differences, of the order of a few tens of nanometers, in the thickness of the layers composing the two beam splitters.

Conversely, the sharp peaks observed around 700 and above $1000 \mathrm{~cm}^{-1}$ originate from absorption bands in the Mylar substrate and impact both the amplitude and phase of the measurements.

The minimum in interferometric efficiency near $1500 \mathrm{~cm}^{-1}$ that is due to the periodical characteristic of multilayer beam splitters is also correctly modeled. Actually the minimum appears split in two due to the small differences in thickness between the two beam splitters mentioned above.

The opposite-facing beam splitter configuration, providing the best optical path compensation, is the one used in the REFIR-PAD instrument. This configuration gives, for each (a) Hot blackbody source $\left(\mathrm{T}_{\mathrm{M}}=353 \mathrm{~K}\right) \quad \mathrm{T}_{\mathrm{R}}=302 \mathrm{~K}, \mathrm{~T}_{\mathrm{BS}}=302 \mathrm{~K}$
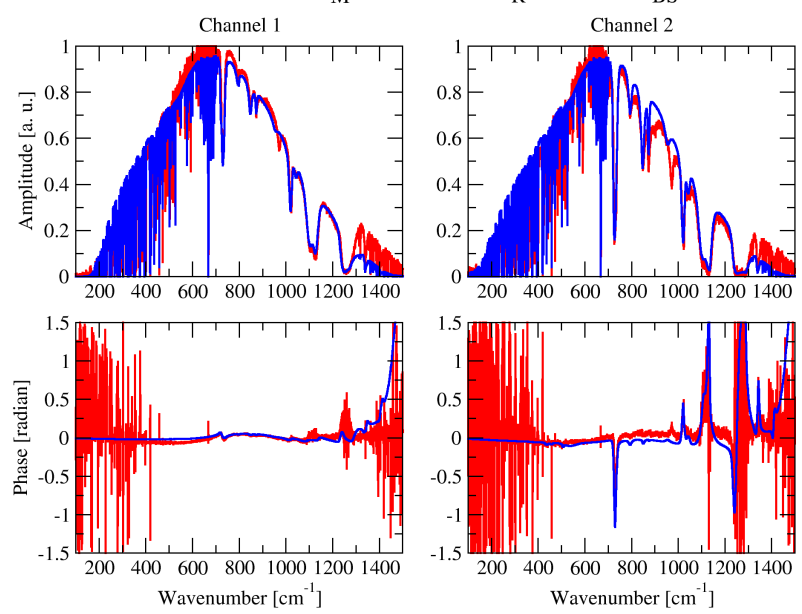

(b) Hot blackbody source $\left(\mathrm{T}_{\mathrm{M}}=353 \mathrm{~K}\right)$

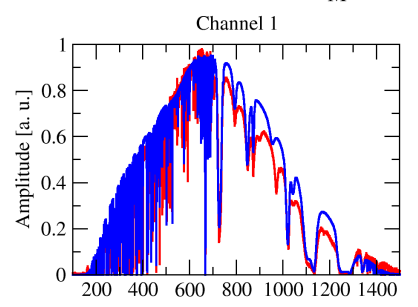

K) $\mathrm{T}_{\mathrm{R}}=302 \mathrm{~K}, \mathrm{~T}_{\mathrm{BS}}=302 \mathrm{~K}$
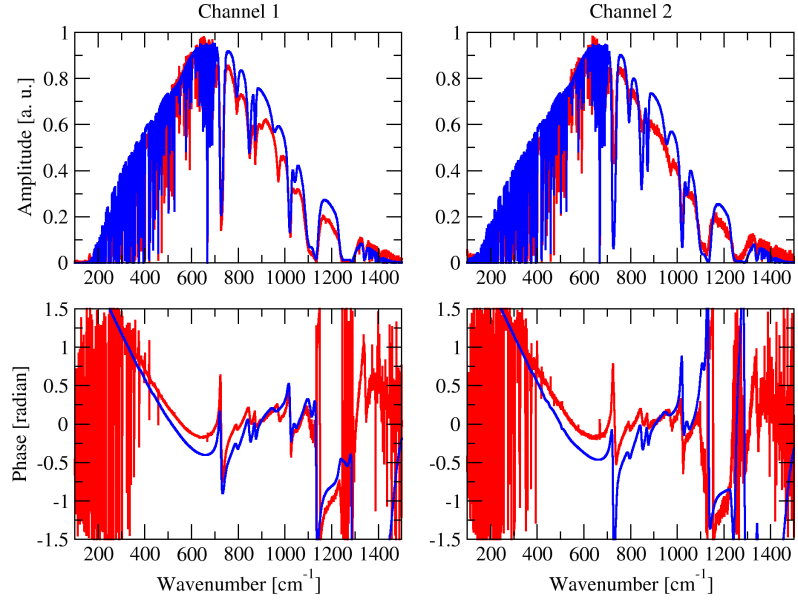

Figure 11. Simulation of the REFIR-PAD instrumental response function for different beam splitter setups (blue line). Compensated (a) and unbalanced (b) configurations are shown. Laboratory measurements (red line) are compared with simulation outputs for the corresponding configuration.

interferometer input, an output with almost complete compensation (channel 1 in Fig. 11) and one with at least partial compensation.

\section{Level 1 products}

The main data product of the REFIR-PAD spectroradiometer is the calibrated atmospheric emitted radiance integrated in the field of view of the instrument (a cone with an aperture of about $10^{\circ}$ ) and spectrally resolved with a $0.4 \mathrm{~cm}^{-1}$ resolution in the $100-1500 \mathrm{~cm}^{-1}$ range. The calibration procedure, described in detail in Bianchini and Palchetti (2008), follows the complex calibration described by Revercomb et al. (1988). The onboard RBB sources are simulated using a specific mathematical model shown in Palchetti et al. (2008). 
REFIR-PAD Dome C 1/3/2018, sequence no. 3, channel 1

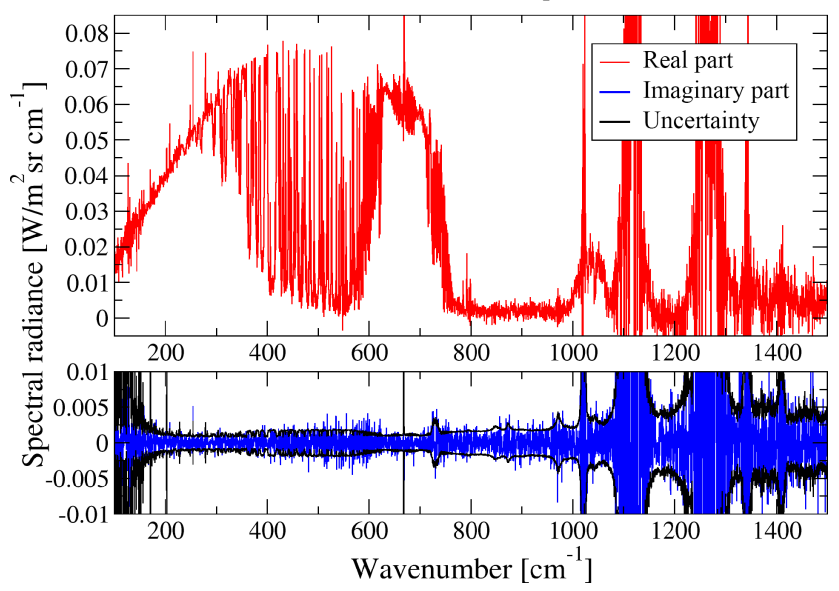

Figure 12. Real (red line), imaginary (blue line), and noise (black) for a typical zenith-looking calibrated spectrum.

Figure 12 shows a typical calibrated spectrum of the DLR (red line) acquired at Dome $\mathrm{C}$ with an integration time of $5 \mathrm{~min}$. The residual imaginary part of the spectrum, after calibration, is also shown (blue line), which is comparable with the estimated noise (black line) as expected.

The REFIR-PAD spectroradiometer has been operated in several campaigns in different environments (tropical, midlatitude, polar), and at different working altitudes, from about sea level to over $5000 \mathrm{~m}$ a.s.l. (Bianchini et al., 2007; Bhawar et al., 2008; Turner et al., 2012). Table 1 shows a list of the campaigns with some information about the available datasets. Of particular importance is the dataset acquired at Dome C, Antarctica (about 3200 m a.s.1.), where the instrument has been acquiring spectrally resolved DLR in all-sky conditions since the end of 2011 (Palchetti et al., 2015).

In Fig. 13, top panel, a set of calibrated spectra acquired in different atmospheric conditions is shown. Each spectrum corresponds to an average of about $6 \mathrm{~h}$ of measurement in clear sky conditions. The measurements span about 2 orders of magnitude in terms of atmospheric total PWV.

The REFIR-PAD instrument has also been operated in nadir-looking observation mode from a stratospheric balloon platform (Palchetti et al., 2006), obtaining atmospheric emission spectra from a $38 \mathrm{~km}$ altitude, thus comparable, for practical purposes, to the top-of-atmosphere condition. In Fig. 13, bottom panel, a comparison between the topof-atmosphere spectrum acquired during the flight and a ground-based zenith-looking measurement performed during the RHUBC-II campaign is shown (Turner et al., 2012).

The measured spectral range includes almost all of the thermal emission from the Earth's atmosphere. If we consider the FIR region $\left(200-667 \mathrm{~cm}^{-1}\right)$, that is the main scientific target of the REFIR-PAD instrument, using the radiometric accuracy figures provided in Sect. 6 and the spectra shown in Fig. 13, we obtain a relative uncertainty in the mea-
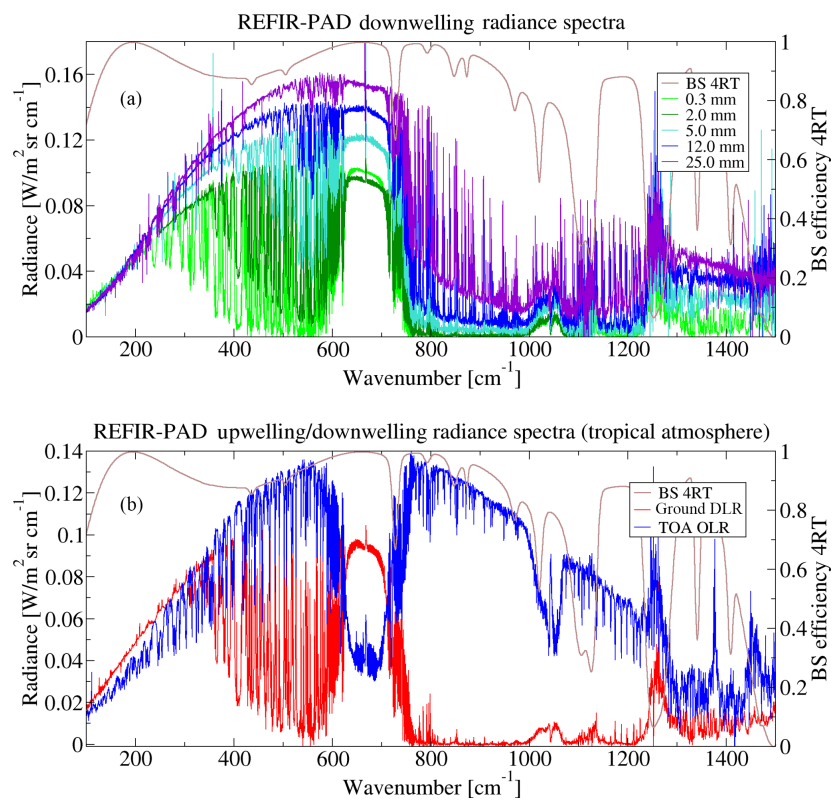

Figure 13. REFIR-PAD level 1 data products. (a) Average zenithlooking spectra corresponding to about $6 \mathrm{~h}$ of data acquisition obtained in different atmospheric humidity conditions, spanning about 2 orders of magnitude in terms of total precipitable water vapor (PWV). The beam splitter efficiency curve is also shown to explain noise bands. (b) High-altitude ground-based zenith-looking spectrum (Cerro Toco, Chile) compared with a top-of-atmosphere nadir-looking measurement from a stratospheric balloon (Teresina, Brazil).

surement of the total radiance that lies between $0.7 \%$ and $2 \%$.

\section{Level 2 products}

The REFIR-PAD level 1 data products can provide plenty of information not only on the radiative properties of the atmosphere, but also on its structure and composition. To perform the retrieval of these variables, a software package has been developed (Bianchini et al., 2011) that is based on the Line-By-Line Radiative Transfer Model (LBLRTM) (Clough et al., 2005) and the MINUIT minimization routines, part of CERNlibs.

The software retrieved temperature and water vapor content profiles on separate vertical grids, together with extra parameters like columnar amounts of minor species, cloud optical thickness, and instrumental parameters such as wavenumber calibration shift and line shape coefficient $\alpha$. The spectral range used in the process is a subset of the full REFIR-PAD spectral range, typically $350-850 \mathrm{~cm}^{-1}$, even if some adjustments to the low-wavenumber end can be made according to the observed PWV ranges.

In detail the retrieval code makes use of the subroutine MIGRAD, which is based on the Davidon-Fletcher-Powell 
Table 1. Data available from the measurement campaigns performed by the REFIR-PAD instrument. Integration time corresponds to the actual acquisition time in zenith-looking mode used to produce a single spectrum, while the repetition rate accounts for the total duration of the acquisition including calibrations and system overhead.

\begin{tabular}{lllrrrr}
\hline & Date & $\begin{array}{l}\text { Duration } \\
\text { (UTC) }\end{array}$ & $\begin{array}{r}\text { Int. time } \\
(\mathrm{min})\end{array}$ & $\begin{array}{r}\text { Rep. rate } \\
(\mathrm{min})\end{array}$ & $\begin{array}{r}\text { Spectral range } \\
\left(\mathrm{cm}^{-1}\right)\end{array}$ & $\begin{array}{r}\text { Resolution } \\
\left(\mathrm{cm}^{-1}\right)\end{array}$ \\
\hline Teresina, Brazil & 30 Jun 2005 & $08: 05-15: 48$ & 6.4 & 10.4 & $100-1100$ & 0.475 \\
Monte Morello, Italy & 6 Feb 2006 & $16: 26-17: 58$ & 5.1 & 7.7 & $350-850$ & 0.5 \\
Monte Gomito, Italy & 13-14 Mar 2006 & 16:20-09:30 (+1) & $6.1 / 9.9$ & $9.2 / 15.7$ & $350-1100$ & 0.5 \\
Testa Grigia, Italy & 4-13 Mar 2007 & 6 days & 5.1 & 11.0 & $240-1400$ & 0.5 \\
Breuil-Cervinia, Italy & 15 Mar 2007 & 15:14-23:09 & 5.1 & 11.0 & $350-1400$ & 0.5 \\
Pagosa Springs, USA & 22-29 Apr 2009 & 6 days & 5.1 & 11.0 & $350-1400$ & 0.5 \\
Cerro Toco, Chile & 21 Aug-24 Oct 2009 & 37 days & 5.1 & 11.0 & $100-1500$ & 0.5 \\
Testa Grigia, Italy & 9-11 Mar 2011 & 3 days & 5.1 & 11.0 & $240-1400$ & 0.25 \\
Dome C, Antarctica & since 21 Dec 2011 & permanent & $6.4 / 5.5$ & $14.1 / 11.9$ & $100-1500$ & 0.4 \\
\hline
\end{tabular}

(DFP) algorithm, to minimize the chi-square cost function given by

$\chi^{2}=(\boldsymbol{y}-\mathbf{F}(\boldsymbol{x}))^{T} \mathbf{S}_{y}^{-1}(\boldsymbol{y}-\mathbf{F}(\boldsymbol{x}))$,

where $\boldsymbol{y}$ and $\boldsymbol{x}$ are the vector of the measurements and the state of the atmosphere, respectively; $\mathbf{F}$ is the forward model (LBLRTM version 12.2 in our case); and $\mathbf{S}_{y}$ is the diagonal variance-covariance matrix for the measurements. The DFP algorithm is a quasi-Newton method that does not require the calculation of the jacobians at each iteration but uses an approximated form. This algorithm updates the inverse Hessian matrix calculating the derivatives just at the first step and then using the iterative formula shown above. The same fitting approach that was applied in previous work (Bianchini et al., 2011) was used in this paper. No a priori information was assumed as regularly done in a Bayesian approach, such as optimal estimation, and the initial guess is represented by a local monthly climatology, obtained averaging over a set of radiosoundings performed daily at Dome C. Since no a priori information was used to constrain the solution and no regularization was introduced, to avoid the oscillation effects due to the ill conditioning of the problem, this approach requires us to limit the number of retrieved parameters; hence the number of fitted levels for both water vapor and temperature profiles is equal to the number of degrees of freedom (DOFs). The DOFs were derived from a preliminary study performed through singular value decomposition of the Hessian matrix, which includes jacobians and the measurement noise.

A typical retrieval operates on a four-point grid for temperature and a five-point grid for water vapor, with retrieval levels chosen on the basis of an analysis of the jacobians of the selected variables. In Fig. 14 the typical result of a fitting process is shown.

Even in this case adjustments to the retrieval grid can be made according to the observed atmosphere properties: as an example, in the case of Antarctic measurements, the high atmospheric transparency and peculiar vertical structure (com-

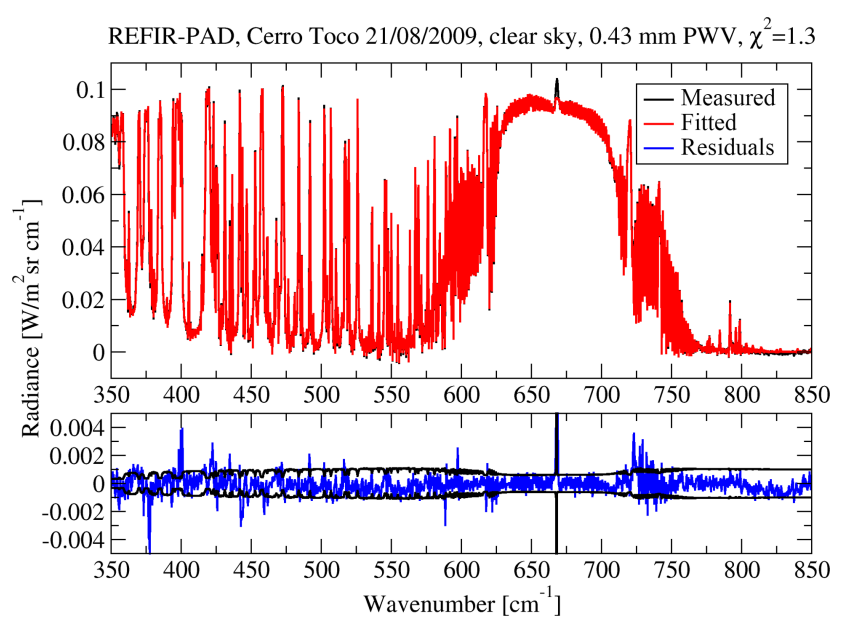

Figure 14. A typical result of the level 2 data analysis process. A single REFIR-PAD measurement is fitted using the LBLRTM forward model and the MINUIT minimization routines. Fitting residuals are compared with the total radiometric uncertainty (black line) in the bottom panel.

ing also from the perturbating effect given by the presence of the shelter in which the instrument is installed) also allow for a retrieval of a five-point profile for temperature.

The vertical profiles of temperature and humidity obtained from the analysis of the set of zenith-looking measurement shown in Fig. 13, top panel, are presented in Fig. 15. These results show how the process can operate in a very wide range of atmospheric conditions.

In order to better reflect the atmospheric modeling performed by LBLRTM, the fitted profiles are shown as histograms following the layering structure adopted in the forward model. The logarithmic scale adopted for the representation of the vertical profile reflects the logarithmic spacing used in the layering, which derives from the decrease in vertical resolution with height that is inherent in the zenithlooking vertical sounding geometry. 


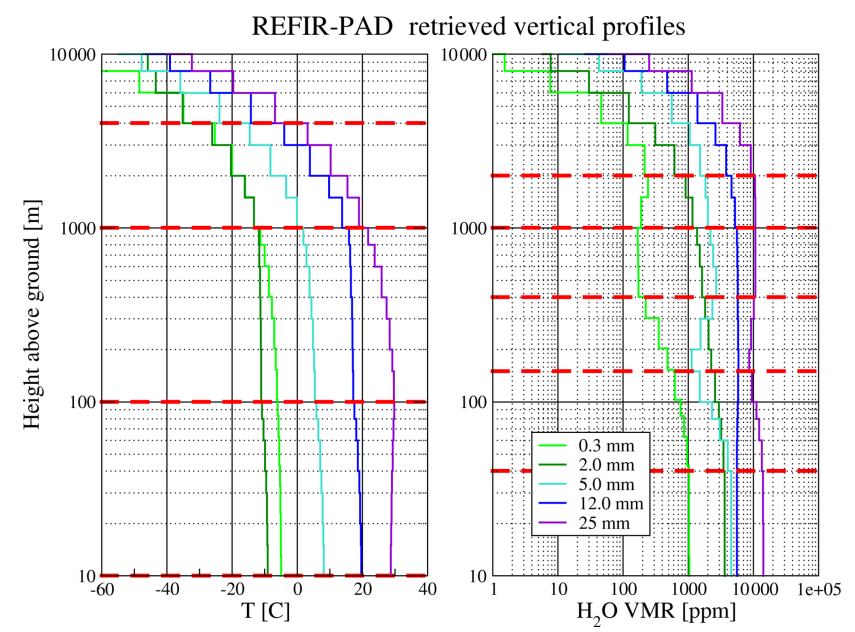

Figure 15. Vertical temperature and water vapor profiles obtained from the spectra in Fig. 13a, through the level 2 data analysis process. Red dashed lines show the selected fitting layers for temperature and water vapor.

A better visualization of the products of the retrieval process can be obtained by plotting the profiles vs. time as color maps. In Fig. 16 the temperature and water vapor maps obtained for the September-December period in 2017 are shown. Note that due to the installation of the instrument inside of a heated shelter, with a $2 \mathrm{~m}$ chimney connecting it to the outside air, the first meters of the retrieved profiles correspond to the air inside the shelter.

The profile maps presented in Fig. 16 show the transition to Antarctic summer cycle, with the onset of a diurnal cycle for the temperature inversion, that can be correctly resolved and characterized with the $12 \mathrm{~min}$ repetition rate of REFIRPAD measurements.

The PWV is also provided as a level 2 data product. The accuracy in the determination of the PWV depends on the atmospheric conditions (total amount of water, presence of clouds) and ranges from $10 \%$ to $20 \%$ in the extremely dry conditions found in Antarctica to about $5 \%$ in the midlatitude atmosphere. Accuracy on the total PWV has been estimated through the error on water vapor column fitting and validated with a microwave radiometer (Fiorucci et al., 2008; Bianchini et al., 2011).

The columnar amount of other tropospheric minor species with spectral lines in the REFIR-PAD measurement range can also be retrieved. For example, nitrous oxide is obtained by adding an extra fit parameter that rescales the vertical $\mathrm{N}_{2} \mathrm{O}$ profile in the temperature and water vapor fitting process, making use of the $589 \mathrm{~cm}^{-1}$ spectral band. In Fig. 17, bottom panel, a time series of the retrieved $\mathrm{N}_{2} \mathrm{O}$ obtained from measurements performed in the September 2017-April 2018 period is shown.

It should be noted that a similar approach could also be applied to methane, provided a suitable spectral window con-

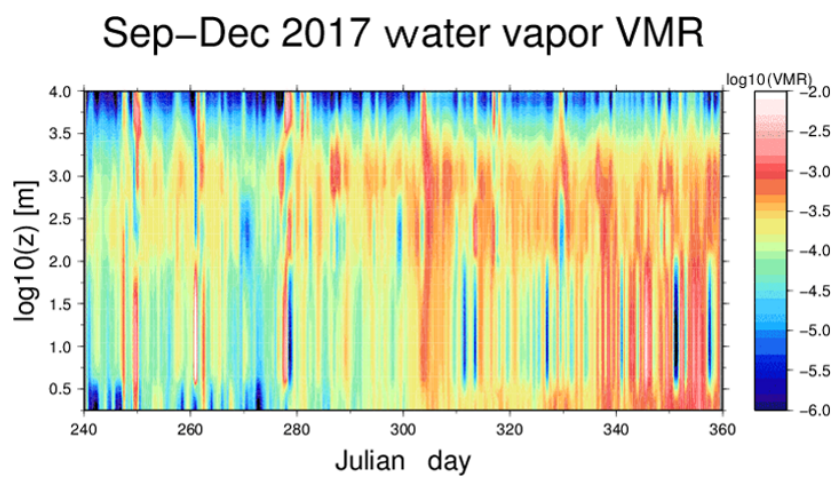

Sep-Dec 2017 temperature

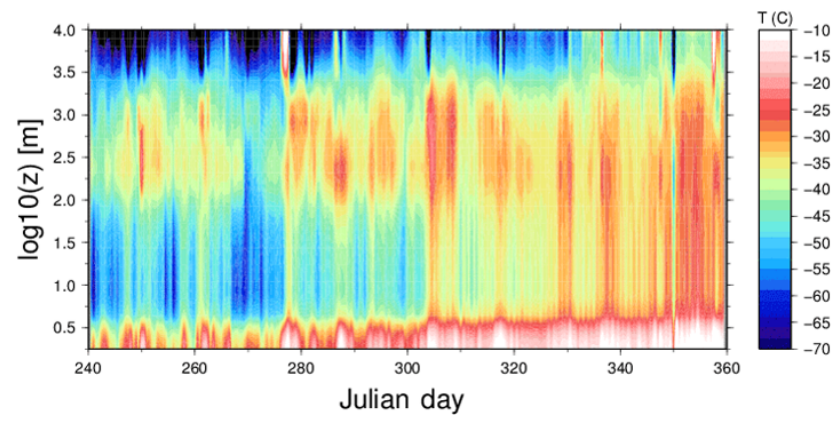

Figure 16. Vertical water vapor and temperature profile maps covering the September-December 2017 period. The warmer layer in the first meters corresponds to the optical path inside of the shelter and of the measurement chimney.

taining methane absorption features is added to the retrieval range. However, the main methane absorption feature overlaps with the absorption bands of the Mylar beam splitter substrate, so a different beam splitter design (e.g., based on polypropylene) would be needed for an efficient methane total column retrieval.

A different consideration must be made for ozone column retrieval: while a strong ozone emission band is present in the REFIR-PAD operating spectral interval, most of the ozone lies in the stratosphere where the temperature retrieval, mainly relying on the carbon dioxide $\nu_{2}$ band, has no sensitivity. Thus to correctly interpret the emitted radiance due to the ozone band, stratospheric temperatures must be provided as an external input. This can be done through radiosounding or an auxiliary sensor such as a stratospheric Raman lidar (Bianchini et al., 2014).

The ozone retrieval process makes use of the 920 $1070 \mathrm{~cm}^{-1}$ spectral range. The retrieval grid, obtained through Jacobian analysis as in the case of temperature and water vapor retrieval, features three fitted levels in the 12 $24 \mathrm{~km}$ altitude range.

In Fig. 17, top panel, ozone columnar amounts obtained in the September 2017-April 2018 period, together with the available NOAA OMI/OMPS ozone time series data (http://www.esrl.noaa.gov/gmd/grad/neubrew/ 


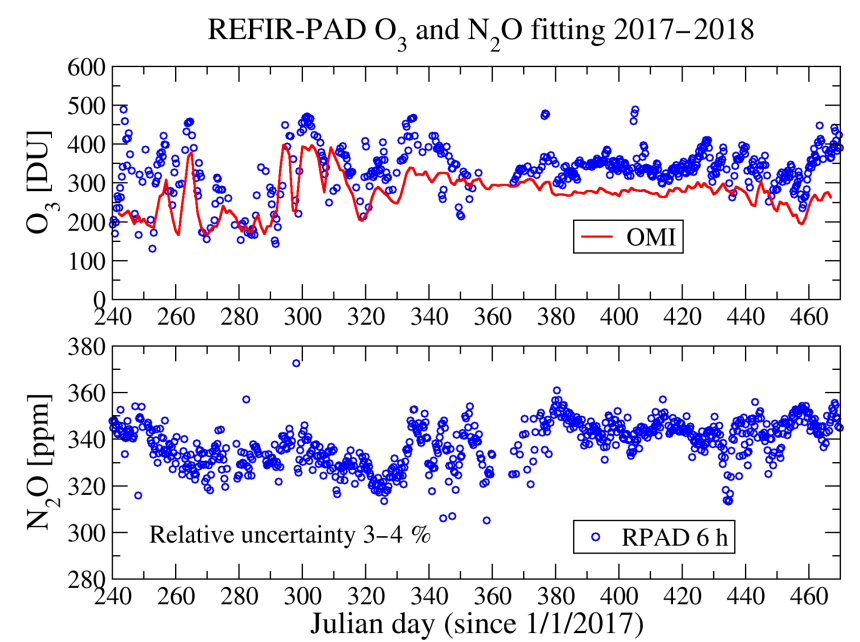

Figure 17. Time series of ozone and nitrous oxide columnar values retrieved from the REFIR-PAD measurements acquired in the September 2017-April 2018 period. For reference, corresponding OMI measurements over the Dome $\mathrm{C}$ region pixel are also shown.

SatO3DataTimeSeries.jsp, last access: 7 October 2018), calculated for the ground pixel corresponding to Concordia station, are shown.

While a noticeable offset in ozone data is present and needs to be investigated further, the temporal variability is in good agreement with the satellite data, and the vertical variability observed in the retrieved three-point profile shows a good correlation with the rapid variations in the columnar amounts. This can be explained with the fact that Dome C lays on the edge of the polar vortex region, so that it can enter and exit the vortex region depending on atmospheric transport.

\section{Level 2 auxiliary outputs}

The instrumental parameters obtained from the level 2 data analysis process provide a valuable tool to characterize the quality of the measured spectra and the performance of the instrument. Figure 18 shows the result of the analysis of the instrumental parameters in the 2016-2017 period. During these 2 years the REFIR-PAD instrument operated continuously without any significant maintenance.

In December 2015 the laser source was replaced due to a malfunction inducing sudden mode jumps, and a laser frequency calibration was performed. Long-term frequency stability of the laser source in the following 2 years can be evaluated from the laser frequency error $\Delta \sigma$, shown in Fig. 18b.

Laser frequency appears to be subjected to a slow drift that accumulated a total deviation of about 25-30 ppm since the initial calibration.

This behavior derives mostly from laser diode aging since it is not correlated with the instrument temperature (shown in Fig. 18c) as, instead, would be the effects due to the con-

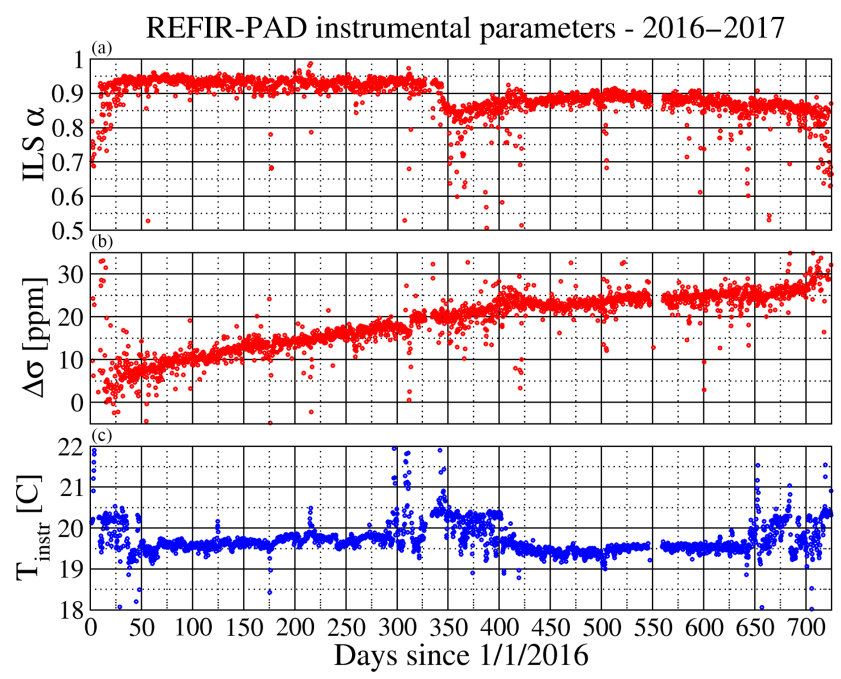

Figure 18. REFIR-PAD instrumental parameters for the 2016-2017 period. (a) ILS coefficient $\alpha$. (b) Laser frequency error $\Delta \sigma$. (c) Instrument temperature.

trol electronics. The observed drift is also about an order of magnitude larger than the temperature drifts estimated using the calculated thermal coefficients (see Sect. 7).

The ILS coefficient $\alpha$ (Fig. 18a) also gives useful insights into the instrument performances in the selected period: it can be seen that during the summer season between 2016 and 2017 (the center part of the plot), larger than usual temperature fluctuation is present. This is due to the higher outside temperatures and also due to personnel working inside of the shelters, which is much more frequent during summer.

The temperature fluctuations impact the ILS coefficient due to thermally induced optical misalignment, which is only partially recovered in the following winter season, so that a yearly optics check and realignment to be performed in summer is desirable even if not mandatory.

\section{Conclusions}

The REFIR-PAD spectroradiometer has proven to be a reliable and versatile tool for the remote sensing of the radiative properties, composition, and thermal structure of the troposphere.

The instrument is capable of providing a wealth of information with a measurement repetition rate of the order of $10 \mathrm{~min}$, fast enough to resolve all the relevant cloud-free atmosphere processes, which is the reference case in this work.

The currently available data products are

- atmospheric emitted radiance spectra in the 100$1500 \mathrm{~cm}^{-1}$ range with $0.4 \mathrm{~cm}^{-1}$ resolution and $0.85 \mathrm{~mW}\left(\mathrm{~m}^{2} \mathrm{srcm}^{-1}\right)^{-1}$ accuracy.

- tropospheric water vapor and temperature vertical profiles with up to five independently fitted points. 
- total columnar precipitable water vapor (PWV) with an accuracy ranging from $5 \%$ to $20 \%$ depending on the total humidity and atmospheric conditions.

- columnar amounts of minor species such as nitrous oxide and ozone.

- cloud optical thickness in the atmospheric transparency window region $\left(800-1200 \mathrm{~cm}^{-1}\right)$.

The instrument operates at room temperature, is fully autonomous, and allows for complete remote control of the configuration parameters; thus it is perfectly suitable for operation in remote and extreme environments, as demonstrated by more than 6 years of continuous operation at the Antarctic station Concordia.

It should be noted that this specific location provides itself a unique dataset since no similar instruments are operating continuously on the Antarctic continent. Currently the REFIR-PAD instrument is operated in the framework of two different projects funded by the Italian National Antarctic Research Program in the perspective of reaching at least a decade-long measurement time series.

Future outlooks include the development and test of new beam splitter designs to overcome the spectral band limitations posed by the use of a Mylar substrate. The development of polypropylene-based beam splitters is currently in progress and will allow us to fully exploit the $1100-1400 \mathrm{~cm}^{-1}$ spectral region to add new products (e.g., methane) to the currently available ones.

Data availability. Data used in this paper are available from the authors upon request.

Competing interests. The authors declare that they have no conflict of interest.

Acknowledgements. We would like to acknowledge the Italian National Antarctic Research Program, Programma Nazionale di Ricerca in Antartide (PNRA) for the funding for the following research programs that have allowed us to perform REFIR-PAD measurements since December 2011 at Concordia station, Antarctica: project PRANA (Proprietà Radiative del vapore Acqueo e delle Nubi in Antartide) 2009/A04.03 2011-2013, project COMPASS (COncordia Multi-Process Atmospheric StudieS) 2013/AC3.01 2013-2016, and the currently active projects DOCTOR (DOme C Tropospheric ObserveR) 2016/AC3.02 and FIRCLOUDS (Far Infrared Radiative Closure Experiment For Antarctic Clouds) 2016/AC3.03.

Edited by: Lars Hoffmann

Reviewed by: two anonymous referees

\section{References}

Bhawar, R., Bianchini, G., Bozzo, A., Calvello, M. R., Cacciani, M., Carlotti, M., Castagnoli, F., Cuomo, V., Di Girolamo, P., Di Iorio, T., Di Liberto, L., di Sarra, A., Esposito, F., Fiocco, G., Fuà, D., Grieco, G., Maestri, T., Masiello, G., Muscari, G., Palchetti, L., Papandrea, E., Pavese, G., Restieri, R., Rizzi, R., Romano, F., Serio, C., Summa, D., Todini, G., and Tosi, E.: Spectrally Resolved Observations of Earth's Emission Spectrum in the $\mathrm{H}_{2} \mathrm{O}$ Rotation Band, Geophys. Res. Lett., 35, L04812, https://doi.org/10.1029/2007GL032207, 2008.

Bianchini, G. and Palchetti, L.: Technical Note: REFIR-PAD level 1 data analysis and performance characterization, Atmos. Chem. Phys., 8, 3817-3826, https://doi.org/10.5194/acp-8-3817-2008, 2008.

Bianchini, G. and Raspollini, P.: Characterization of instrumental line shape distortions due to path difference dependent phase errors in a Fourier transform spectrometer, Infr. Phys. Techn., 41, 287-292, 2000.

Bianchini, G., Lanfranchi, M., and Cortesi, U.: Flight qualification of a diode laser for path difference determination of a high-resolution Fourier transform spectrometer, Appl. Optics, 39, 962-965, 2000.

Bianchini, G., Cortesi, U., Palchetti, L., and Pascale, E.: SAFIRE/A (spectroscopy of the atmosphere by far-infrared emission - airborne): optimised instrument configuration and new assessment of improved performances, Appl. Optics, 43, 2962-2977, 2004.

Bianchini, G., Boscaleri, A., Carli, B., Mencaraglia, F., Palchetti, L., and Pascale, E.: IBEX (Infrared Balloon Experiment): improved instrumental configuration and assessment of instrument performances, Appl. Optics, 45, 1041-1051, 2006 a.

Bianchini, G., Castagnoli, F., Pellegrini, M., and Palchetti, L.: Frictionless mirror drive for intermediate resolution infrared Fourier transform spectroscopy, Infr. Phys. Techn., 48, 217-222, 2006b.

Bianchini, G., Palchetti, L., Baglioni, A., and Castagnoli, F.: Farinfrared spectrally resolved broadband emission of the atmosphere from Morello and Gomito mountains near Florence, Remote Sensing of Clouds and the Atmosphere XII, edited by: Comerón, A., Picard, R. H., Schäfer, K., Slusser, J. R., and Amodeo, A., Proc. SPIE, 6745, 674515-1-674515-12, 2007.

Bianchini, G., Palchetti, L., and Carli, B.: Vectorial combination of signals in Fourier transform spectroscopy, Infr. Phys. Techn., 52, 19-21, https://doi.org/10.1016/j.infrared.2008.09.004, 2009.

Bianchini, G., Palchetti, L., Muscari, G., Fiorucci, I., Di Girolamo, P., and Di Iorio, T.: Water vapor sounding with the far infrared REFIR-PAD spectroradiometer from a high-altitude groundbased station during the Ecowar campaign, J. Geophys. Res. 116, D02310, https://doi.org/10.1029/2010JD014530, 2011.

Bianchini, G., Argentini, S., Baldi, M., Cairo, F., Calzolari, F., Casasanta, G., Conidi, A., Del Guasta, M., Di Natale, G., Federico, S., Lupi, A., Mazzola, M., De Muro, M., Palchetti, L., Petenko, I., Petkov, B., Snels, M., Trivellone, G., Viola, A., and Viterbini, M.: Concordia Multi-Process Atmospheric Studies (CoMPASs): study of the vertical structure of the Antarctic atmosphere with a synergy of different remote sensing techniques, EGU General Assembly Conference Abstracts, 16, 7782, 2014.

Brindley H. E. and Harries, J. E.: The impact of far I. R. absorption on clear sky greenhouse forcing: sensitivity studies at high spectral resolution, J. Quant. Spectrosc. Ra., 60, 151-180, 1998. 
Carli, B., Barbis, A., Harries, J. E., and Palchetti, L.: Design of an efficient broad band far infrared FT spectrometer, Appl. Optics, 38, 3945-3950, 1999a.

Carli, B., Palchetti, L., and Raspollini, P.: Effect of beam splitter emission in Fourier-transform spectroscopy, Appl. Optics, 38, 7475-7480, 1999b.

Clerbaux, N., Dewitte, S., Gonzalez, L., Bertrand, C., Nicula, B., and Ipe, A.: Outgoing longwave flux estimation: improvement of angular modelling using spectral information, Remote Sens. Environ., 85, 389-395, 2003.

Clough, S. A., Shephard, M. W., Mlawer, E. J., Delamere, J. S., Iacono, M. J., Cady-Pereira, K., Boukabara, S., and Brown, P. D.: Atmospheric radiative transfer modeling: a summary of the AER codes: Short communication, J. Quant. Spectrosc. Ra., 91, 233244, 2005.

Fiorucci, I., Muscari, G., Bianchi, C., Di Girolamo, P., Esposito, F., Grieco, G., Summa, D., Bianchini, G., Palchetti, L., Cacciani, M., Di Iorio, T., Pavese, G., Cimini, D., and de Zafra, R. L.: Measurements of low amounts of precipitable water vapor by mm-wave spectroscopy: an intercomparison with radiosonde, Raman Lidar and FTIR data, J. Geophys. Res., 113, D14314, https://doi.org/10.1029/2008JD009831, 2008.

Gero, P. J. and Turner, D. D.: Long-Term Trends in Downwelling Spectral Infrared Radiance over the U.S. Southern Great Plains, J. Climate, 24, 4831-4843, https://doi.org/10.1175/2011JCLI4210.1, 2011.

Green, P. D., Newman, S. M., Beeby, R. J., Murray, J. E., Pickering, J. C., and Harries, J. E.: Recent advances in measurement of the water vapour continuum in the farinfrared spectral region, Philos. T. R. Soc. A, 370, 2637-2655, https://doi.org/10.1098/rsta.2011.0263, 2012.

Harries, J. E., Carli, B., Rizzi, R., Serio, C., Mlynczak, M., Palchetti, L., Maestri, T., Brindley, H., and Masiello, G.: The Far-Infrared Earth, Rev. Geophys., 46, RG4004, https://doi.org/10.1029/2007RG000233, 2008.

Huang, Y.: A Simulated Climatology of Spectrally Decomposed Atmospheric Infrared Radiation, J. Climate, 26, 1702-1715, https://doi.org/10.1175/JCLI-D-12-00438.1, 2013.

Huang, Y., Ramaswamy, V., Huang, X., Fu, Q., and Bardeen, C.: A strict test in climate modeling with spectrally resolved radiances: GCM simulation versus AIRS observations, Geophys. Res. Lett., 34, L24707, https://doi.org/10.1029/2007GL031409, 2007.

Loeb, N. G., Kato, S., Loukachine, K., and Manalo-Smith, N.: Angular Distribution Models for Top-of-Atmosphere Radiative Flux Estimation from the Clouds and the Earth's Radiant Energy System Instrument on the Terra Satellite. Part I: Methodology, J. Atmos. Ocean. Tech., 22, 338-351, https://doi.org/10.1175/JTECH1712.1, 2005.

Loeb, N. G., Kato, S., Loukachine, K., Manalo-Smith, N., and Doelling, D. R.: Angular Distribution Models for Top-ofAtmosphere Radiative Flux Estimation from the Clouds and the Earth's Radiant Energy System Instrument on the Terra Satellite. Part II: Validation, J. Atmos. Ocean. Tech., 24, 564-584, https://doi.org/10.1175/JTECH1983.1, 2007.

Mariani, Z., Strong, K., Wolff, M., Rowe, P., Walden, V., Fogal, P. F., Duck, T., Lesins, G., Turner, D. S., Cox, C., Eloranta, E., Drummond, J. R., Roy, C., Turner, D. D., Hudak, D., and Lindenmaier, I. A.: Infrared measurements in the Arctic using two Atmospheric Emitted Radiance Interferometers,
Atmos. Meas. Tech., 5, 329-344, https://doi.org/10.5194/amt-5329-2012, 2012.

Martin D. H. and Puplett, E.: Polarised interferometric spectrometry for the millimetre and submillimetre spectrum, Infr. Phys. Techn., 10, 105-109, 1969.

Mlynczak, M. G., Cageao, R. P., Mast, J. C., Kratz, D. P., Latvakoski, H., and Johnson, D. G.: Observations of downwelling far-infrared emission at Table Mountain California made by the FIRST instrument, J. Quant. Spectrosc. Ra., 170, 90-105, 2016.

Ohmura, A., Dutton, E. G., Forgan, B., Frohlich, C., Gilgen, H., Hegner, H., Heimo, A., Konig-Langlo, G., McArthur, B., Miiller, G., Philipona, R., Pinker, R., Whitlock, C. H., Dehne, K., and Wild, M.: Baseline Surface Radiation Network (BSRN/WCRP): New precision radiometry for climate research, B. Am. Meteorol. Soc., 79, 2115-2136, 1998.

Palchetti, L., Barbis, A., Harries, J. E., and Lastrucci, D.: Design and mathematical modelling of the space-borne farinfrared Fourier transform spectrometer for REFIR experiment, Infr. Phys. Techn., 40, 367-377, 1999.

Palchetti, L., Bianchini, G., Castagnoli, F., Carli, B., Serio, C., Esposito, F., Cuomo, V., Rizzi, R., and Maestri, T.: Breadboard of a Fourier-transform spectrometer for the Radiation Explorer in the Far Infrared atmospheric mission, Appl. Optics, 44, 2870-2878, 2005.

Palchetti, L., Belotti, C., Palchetti, L., Belotti, C., Bianchini, G., Castagnoli, F., Carli, B., Cortesi, U., Pellegrini, M., CamyPeyret, C., Jeseck, P., and Té, Y.: Technical note: First spectral measurement of the Earth's upwelling emission using an uncooled wideband Fourier transform spectrometer, Atmos. Chem. Phys., 6, 5025-5030, https://doi.org/10.5194/acp-6-5025-2006, 2006.

Palchetti, L., Bianchini, G., and Castagnoli, F.: Design and characterisation of black-body sources for infrared wide-band Fourier transform spectroscopy, Infr. Phys. Techn., 51, 207-215, https://doi.org/10.1016/j.infrared.2007.06.001, 2008.

Palchetti, L., Bianchini, G., Di Natale, G., and Del Guasta, M.: Far infrared radiative properties of water vapor and clouds in Antarctica, B. Am. Meteorol. Soc., 96, 1505-1518, https://doi.org/10.1175/BAMS-D-13-00286.1, 2015.

Palchetti, L., Lanconelli, C., Bianchini, G., and Di Natale, G.: Spectral characterization of the surface longwave radiation over the East Antarctic Plateau, AIP Conf. Proc., 1810, 100005-1100005-4, 2017.

Reichert, A. and Sussmann, R.: The Zugspitze radiative closure experiment for quantifying water vapor absorption over the terrestrial and solar infrared - Part 3: Quantification of the mid- and near-infrared water vapor continuum in the 2500 to $7800 \mathrm{~cm}^{-1}$ spectral range under atmospheric conditions, Atmos. Chem. Phys., 16, 11671-11686, https://doi.org/10.5194/acp-16-116712016, 2016.

Revercomb, H. E., Buijs, H., Howell, H. B., LaPorte, D. D., Smith, W. L., and Sromovsky, L. A.: Radiometric calibration of IR Fourier transform spectrometers: solution to a problem with the High-Resolution Interferometer Sounder, Appl. Optics, 27, 3210-3218, 1988.

Ridolfi, M., Carli, B., Carlotti, M., von Clarmann, T., Dinelli, B. M., Dudhia, A., Flaud, J.-M., Höpfner, M., Morris, P. E., Raspollini, P., Stiller, G., and Wells, R. J.: Optimized forward model and retrieval scheme for MIPAS near-real-time dataprocessing, Appl. 
Optics, 39, 1323-1340, https://doi.org/10.1364/AO.39.001323, 2000

Rizzi, R., Palchetti, L., Carli, B., Bonsignori, R., Harries, J. E., Leotin, J., Peskett, S., Serio, C., and Sutera, A.: Feasibility study of the space-borne Radiation Explorer in the Far InfraRed (REFIR), in Optical Spectroscopic Techniques, Remote Sensing and Instrumentation for Atmospheric and Space Research IV, edited by: Larar, A. M. and Mlynczak, M. G., Proc. SPIE, 4485, 202209, 2002.

Shi, S.-C., Paine, S., Yao, Q.-J., Lin, Z.-H., Li, X.-X., Duan, W.-Y., Matsuo, H., Zhang, Q., Yang, J., Ashley, M. C. B., Shang, Z., and Hu, Z.-W.: Terahertz and far-infrared windows opened at Dome A in Antarctica, Nature Astronomy, 1, 0001, https://doi.org/10.1038/s41550-016-0001, 2016.

Sinha A. and Harries, J. E.: Water vapor greenhouse trapping: The role of the far infrared absorption, Geophys. Res. Lett., 22, $2147-$ 2150, 1995.

Smith, W. L., Feltz, W. F., Knuteson, R. O., Revercomb, H. E., Woolf, H. M., and Howell, H. B.: The Retrieval of Planetary Boundary Layer Structure Using GroundBased Infrared Spectral Radiance Measurements, J. Atmos. Ocean. Tech., 16, 323-333, https://doi.org/10.1175/15200426(1999)016<0323:TROPBL>2.0.CO;2, 1999.

Stephens, G. L., Li, J., Wild, M., Clayson, C. A., Loeb, N., Kato, S., L'Ecuyer, T., Stackhouse Jr., P. W., Lebsock, M., and Andrews, T.: An update on Earth's energy balance in light of the latest global observations, Nat. Geosci., 5, 691-696, https://doi.org/10.1038/ngeo1580, 2012.

Town, M. S., Walden, V. P., and Warren, S. G.: Spectral and Broadband Longwave Downwelling Radiative Fluxes, Cloud Radiative Forcing, and Fractional Cloud Cover over the South Pole, J. Climate, 18, 4235-4252, https://doi.org/10.1175/JCLI3525.1, 2005.
Turner, D. D., Tobin, D. C., Clough, S. A., Brown, P. D., Ellingson, R. G., Mlawer, E. J., Knuteson, R. O., Revercomb, H. E., Shippert, T. R., Smith, W. L., and Shephard, M. W.: The QME AERI LBLRTM: A Closure Experiment for Downwelling High Spectral Resolution Infrared Radiance, J. Atmos. Sci., 61, 2657-2675, https://doi.org/10.1175/JAS3300.1, 2004.

Turner, D. D., Mlawer, E. J., Bianchini, G., Cadeddu, M. P., Crewell, S., Delamere, J. S., Knuteson, R. O., Maschwitz, G., Mlynzcak, M., Paine, S., Palchetti, L., and Tobin, D. C.: Ground-based high spectral resolution observations of the entire terrestrial spectrum under extremely dry conditions, Geophys. Res. Lett., 39, L10801, https://doi.org/10.1029/2012GL051542, 2012.

Turner, E. C., Lee, H.-T., and Tett, S. F. B.: Using IASI to simulate the total spectrum of outgoing long-wave radiances, Atmos. Chem. Phys., 15, 6561-6575, https://doi.org/10.5194/acp15-6561-2015, 2015.

Vanasse, G. A. and Sakai, H., VII Fourier Spectroscopy, Progress in Optics, edited by: Wolf, E., 6, 259-330, 1967.

Wild, M., Folini, D., Schär, C., Loeb, N., Dutton, E. G., and KönigLanglo, G.: The global energy balance from a surface perspective, Clim. Dynam., 40, 3107-3134, 2013.

Walden, V., Town, M., Halter, B., and Storey, J.: First measurements of the infrared sky brightness at Dome C, Antarctica, Publications of the Astronomical Society of the Pacific, 117, 300, 2005.

Walden, V. P., Roth, W. L., Stone, R. S., and Halter, B.: Radiometric validation of the Atmospheric Infrared Sounder over the Antarctic Plateau, J. Geophys. Res.-Atmos., 111, D09S03, https://doi.org/10.1029/2005JD006357, 2006. 